D. Comenetz

Nagoya Math. J.

Vol. 82 (1981), 1-26

\title{
TWO ALGEBRAIC DEFORMATIONS OF A K3 SURFACE
}

\author{
DANIEL COMENETZ
}

\section{Introduction}

Let $X$ be a nonsingular algebraic $K 3$ surface carrying a nonsingular hyperelliptic curve of genus 3 and no rational curves. Our purpose is to study two algebraic deformations of $X$, viz. one specialization and one generalization. We assume the characteristic $\neq 2$. The generalization of $X$ is a nonsingular quartic surface $Q$ in $\boldsymbol{P}^{3}$ : we wish to show in $\S 1$ that there is an irreducible algebraic family of surfaces over the affine line, in which $X$ is a member and in which $Q$ is a general member. The specialization of $X$ is a surface $Y$ having a birational model which is a ramified double cover of a quadric cone in $\boldsymbol{P}^{3}$. It has been observed [19] that such a specialization exists; what we propose to show is that there are two different ways to get to it, that is there are two non-isomorphic irreducible algebraic families $\mathscr{S}, \mathscr{S}^{*}$ of surfaces over the affine line, each having a surface $Y$ as a member and having $X$ as a general member. In fact it is shown in $\S 3$ that the "elementary operation" (cf. $\S 2$ ) in the known family $\mathscr{S}$, along a single, nonextending nodal curve $R$ on $Y$ (i.e. $R$ is a non-singular rational curve with self-intersection -2 on $Y$ ), exists algebraically-it is always defined analytically, [3], [9]-and defines a birational transformation $\eta$ of $\mathscr{S}$. The image $\mathscr{S}^{*}=\eta(\mathscr{S})$ is an algebraic family over the affine line, not isomorphic to $\mathscr{S}$ but having the same members. We remark that, while $\mathscr{S}$ and $\mathscr{S}^{*}$ can be regarded separately as families of polarized surfaces, in the sense of [13], the birational correspondence $\eta$ between them does not respect any structure of polarization of general members; hence there is no conflict with Theorem 2 in [15], even though $\eta$ induces an isomorphism between general members $X$ but the graph of $\eta$ does not specialize to the graph of an isomorphism between special members $Y$. To establish the existence of $\eta$ we apply a theorem

Received July 5, 1976.

Revised December 18, 1978. 
of Lascu [12] about algebraic blowing-down along ruled divisors.

This result, existence of $\eta$, is in the spirit of part of the work [3] of Burns and Rapoport where (besides the main theorem) it is shown that elementary operations are "the main reason for the phenomenon of nonseparatedness in the moduli of unpolarized non-ruled algebraic surfaces over $C . "$

In $\S 2$ and in the Appendix we discuss algebraic elementary operations a little more generally than is required for our example. Elementary operations are always defined in the analytic case, as mentioned above, but the question of existence in the algebraic case seems delicate.

The author wishes to express his sincere thanks to Igor Dolgachev for several helpful conversations.

\section{§0. Preliminaries}

0.1. Here we fix notation for certain surfaces which will be used frequently. In the first place we recall two well known ruled surfaces, denoted $F_{0}$ and $F_{2}$. Let $\mathcal{O}=\mathcal{O}_{\boldsymbol{P} \mathbf{1}} . \quad F_{0}$ is, equivalently, $\boldsymbol{P}^{1} \times \boldsymbol{P}^{1} ; \boldsymbol{P}(\mathcal{O}(-1)$ $\oplus \mathcal{O}(-1)$ ) (or $\boldsymbol{P}\left(\mathcal{O}(n) \oplus \mathcal{O}(n)\right.$ ), any $n$ ); a nonsingular quadric surface in $\boldsymbol{P}^{3}$. $F_{2}$ is, equivalently, 2 copies $U_{1}, U_{2}$ of $P^{1} \times A^{1}$ with coordinates $\left(\zeta_{i}, z_{i}\right)$ $i=1$, 2, identified by $\zeta_{2}=z_{1}^{2} \zeta_{1}, z_{2}=z_{1}^{-1} ; P(\mathcal{O}(-2) \oplus \mathcal{O})$; the minimal desingularization of a quadric cone in $P^{3} \quad F_{0}$ carries 2 linear pencils of lines, or rulings, denoted by $\langle\ell\rangle$ and $\left\langle\ell^{\prime}\right\rangle$, where $\ell$ is a line in the pencil $\langle\ell\rangle . \quad F_{2}$ carries a single ruling, denoted $\langle\ell\rangle$, and a nonsingular rational nodal curve $b$ with $b^{(2)}=-2$. $\Delta$ denotes a curve on either surface, a nonsingular member of $\Lambda\left(\ell+\ell^{\prime}\right)^{*}$ in the case of $F_{0}$, and of $\Lambda(2 \ell+b)$ on $F_{2}-\Delta$ is a veritable conic; $\Delta$ on $F_{0}$ is called the diagonal; $\Delta^{(2)}=2$ in both cases. Let $\mathscr{N}$ denote, either $\mathcal{O}(-2) \oplus \mathcal{O}$ or $\mathcal{O}(-1) \oplus \mathcal{O}(-1)$; in both cases there is**) an exact sequence $0 \rightarrow \mathcal{O}(-2) \rightarrow \mathscr{N} \rightarrow \mathcal{O} \rightarrow 0$ of sheaves on $\boldsymbol{P}^{1}$, and the sub-invertible-sheaf $\mathcal{O}(-2) \subset \mathscr{N}$ corresponds to $\Delta$ on $\boldsymbol{P}(\mathscr{N})$.

We shall also have to deal with 2 types of $K 3$ surfaces, denoted by $X$ and $Y$. These are nonsingular, finite double coverings of $F_{0}$ and $F_{2}$ respectively, with nonsingular branch curve $B \sim 4 \Delta$. The reader may con-

*) $\Lambda(X)$ denotes the complete linear system of positive divisors linearly equivalent to a divisor $X$, on a variety:

**) In the $2^{\text {nd }}$ case, switch to vector bundles and note that

$$
\left(\begin{array}{ll}
t & 0 \\
0 & t
\end{array}\right)=\left(\begin{array}{rr}
t^{-1} & -1 \\
1 & 0
\end{array}\right)\left(\begin{array}{ll}
t^{2} & t \\
0 & 1
\end{array}\right)\left(\begin{array}{ll}
1 & 0 \\
t & 1
\end{array}\right)^{-1}
$$

where $t$ denotes a non-homogeneous coordinate on $\boldsymbol{P}^{\mathbf{1}}$. 
sult [16], [20], [4], [19] for numerous facts and details as to these surfaces. They can be constructed as follows (see also the review of double covers below).

Fix a nonsingular curve $B$ in the complete linear system $\Lambda(4 \Delta)$ on $F_{n}, n=0$ or 2 . $B$ is irreducible and, in the case of $F_{2}, B \cap b$ is empty. Let $s$ be the section of $\mathcal{O}_{F_{n}}(4 \Delta)$ corresponding to $B$. (We can think of $s$ as being induced on $F_{n}$ in $P^{3}$ by a homogeneous form of degree 4 in $P^{3}$.) Now $s$ defines an $\mathcal{O}_{F_{n}}$-algebra structure $\mathscr{A}_{n}$ in $\mathcal{O}_{F_{n}} \oplus \mathcal{O}_{F_{n}}(-2)$, determined uniquely by choice of $s$, i.e. choice of $B . \quad X$ is then by definition $\operatorname{Spec}_{F_{0}}\left(\mathscr{A}_{0}\right)$ and $Y$ is $\operatorname{Spec}_{F_{2}}\left(\mathscr{A}_{2}\right)$.

Suppose $X$ and $Y$ are so constructed as double covers. Then $X$ carries two complete linear pencils $\langle E\rangle,\left\langle E^{\prime}\right\rangle$ obtained by pulling back $\langle\ell\rangle,\left\langle\ell^{\prime}\right\rangle$ from $F_{0}$; a general member of either pencil is a nonsingular curve of genus 1 , and every member is irreducible (since $B$ is nonsingular). $A$ general member of $\Lambda\left(E+E^{\prime}\right)$ is a nonsingular hyperelliptic curve of genus 3 so these are the $K 3$ surfaces $X$ mentioned in the Introduction. $Y$ carries one similar pencil $\langle E\rangle$ and 2 nodal curves $R, \bar{R}$ with $\pi(R)=b$ $=\pi(\bar{R})$, where $\pi: Y \rightarrow F_{2}$ is the covering map. $R \cap \bar{R}$ is empty since $\pi$ does not ramify over $b$, and $I(R, E)=1=I(\bar{R}, E)$.

Conversely, given a $K 3$ surface $X$ carrying nonsingular curves $E, E^{\prime}$ of genus 1 with $I\left(E, E^{\prime}\right)=2$ and every member of $\langle E\rangle$ and $\left\langle E^{\prime}\right\rangle$ irreducible, the complete linear system $\Lambda\left(E+E^{\prime}\right)$ defines a finite double covering $X \rightarrow F_{0}$ branched over a nonsingular curve $B \sim 4 \Delta$, and a general member of $\Lambda\left(E+E^{\prime}\right)$ is a nonsingular hyperelliptic curve of genus 3 . Under similar assumptions we get a finite double cover $Y \rightarrow F_{2}$ with nonsingular branch using $\Lambda(3 E+R+\bar{R})$ for instance-note that the mapping defined by $\Lambda(2 E+R+\bar{R})$ collapses $R+\bar{R}$ to a point.

In passing we mention the relation between change of branch curve and change of surface. By a marked $K 3$ surface of type $x$ (resp. type $y$ ) is meant a $K 3$ surface $X$ carrying curves $E, E^{\prime}$ (resp. a $K 3$ surface $Y$ carrying curves $E, R, \bar{R}$ ) as above, together with a complete linear system $\Lambda\left(E+E^{\prime}\right)$ (resp. $\Lambda(E+R+\bar{R})$ ) on it. An isomorphism of marked surfaces of given type must by definition preserve the linear system up to numerical equivalence. Then one can show that there is a bijection between the set of classes of nonsingular branch curves $B$ on $F_{0}$ (resp. on $F_{2}$ ) modulo biregular automorphisms of $F_{0}$ (of $F_{2}$ ), and the set of marked $K 3$ surfaces $X$ (resp. Y) modulo isomorphisms of the marked surfaces. We do not 
use this result, but employing it one can "count" 18 moduli for $X, 17$ moduli for $Y$. Of course a nonsingular quartic surface in $\boldsymbol{P}^{3}$ depends on 19 moduli.

As to finite double covers generally, we shall need the following facts. Some references are [25], [4], [8], [19]; we follow the last in particular.

Let $S$ be a nonsingular variety. A finite double cover of $S$ is a variety $X$ and a finite, flat, morphism $p: X \rightarrow S$ of degree 2. An isomorphism $f$ between two such covers $(X, p)$ and $\left(X^{\prime}, p^{\prime}\right)$ must respect the covering morphisms, i.e. we must have $p=p^{\prime} \circ f$.

Suppose $S$ is complete. There is a bijection between the set $\{(X, p)\}$ of finite double covers of $S$, up to isomorphisms, and the set of pairs $\{(D, s)\}$ where $D$ is an effective divisor on $S$ and $s$ is a section of $\mathcal{O}_{S}(2 D)$, up to scalar multiples of $s$, and linear equivalence of $D$ and automorphisms of $S$. Moreover, given $(D, s)$, the divisor $B$ defined by $s=0$ is the branch locus of $p$. $X$ and $B$ are each nonsingular just when the other is.

If $S$ is not complete, $s$ is determined up to elements of $\left(H^{0}\left(S, \mathcal{O}_{S}\right)^{*}\right)^{2}$.

$X$ is handily constructed as follows ([19]; [6] II.1.3). Let $(D, s)$ be given. $s$ defines an $\mathcal{O}_{S}$-linear map $\mathcal{O}_{S}(-D) \otimes \mathcal{O}_{S}(-D) \rightarrow \mathcal{O}_{S}$, that is an $\mathcal{O}_{S^{-}}$ algebra structure $\mathscr{A}$ on the locally free $\mathcal{O}_{S}$-module $\mathcal{O}_{S} \oplus \mathcal{O}_{S}(-D)$ of rank 2 , and the $\mathcal{O}_{S}$-algebra structure is determined uniquely up to $\mathcal{O}_{S}$-algebra isomorphisms by choice of $s$. $X$ is then $\operatorname{Spec}_{S}(\mathscr{A})$ and $p_{*} \mathcal{O}_{X}=\mathscr{A}$.

The idea of this procedure is to find $X$ as a divisor in the line bundle $[D]$, with local equations $w_{i}^{2}=s_{i}$, where $w_{i}$ is a local fibre coordinate in $[D]$ and $s_{i}=0$ is a local equation for $B$, with respect to a suitable open cover $\left\{U_{i}\right\}$ of $S$.

0.2. We sometimes employ notational conventions of [26], particularly in the following situation. Suppose $f: V \rightarrow W$ is a mapping of varietiesnot necessarily a morphism-and $X$ is a cycle on $V$, (The support of $X$ is simple on $V$ by definition of a cycle.) Let $\Gamma \subset V \times W$ be the graph of $f$ and suppose $\Gamma$ is simple on $V \times W$. Then $f(X)$ denotes the algebraic image of the cycle $X$, defined by $f(X)=p r_{W}[\Gamma \cdot(X \times W)]$ ([26]); when $f$ is a morphism this is the direct image $f_{*}(X)$ ([24], Ch. 5). Moreover, whenever $Y$ is a cycle on $W, f^{-1}(Y)$ denotes the algebraic counter image of the cycle $Y$, defined as $\operatorname{pr}_{V}[\Gamma \cdot(V \times Y)]$; and when $f$ is a morphism and $W$ is nonsingular, this is the reciprocal image $f^{*}(Y)$, that is the alternating sum of cycles of the sheaves $\mathscr{T}_{{ }_{0} r_{i}}\left(\mathcal{O}_{V}, f^{*} \mathcal{O}_{Y}\right)$, cf. [24]. 


\section{$\S 1$. Specialization of a quartic surface in $P^{3}$}

Let a $K 3$ surface $X$ be given as described in (0.1): there is a finite flat morphism $\pi: X \rightarrow F_{0}$ of degree 2, branching over an irreducible nonsingular curve $B$ in the complete linear system $A(4 \Delta)$. We begin with finding a suitable projective model for $X$.

Proposition 1. (a) Suppose $\pi: Z \rightarrow S$ is a finite, flat morphism of degree 2 between a pair of complete nonsingular surfaces $Z, S$. Let $B$ be the branch curve of $\pi$ on $S$ and $D$ a curve on $S$ with $2 D \sim B$. Assume that $D$ is very ample and, when $\varphi=\varphi_{D}$ is the embedding defined by $\Lambda(D)$, $S^{\prime}=\varphi(S)$ is a surface in $\boldsymbol{P}^{N-1}$ on which the linear system cut by quadric hypersurfaces in $\boldsymbol{P}^{N-1}$ is complete. Regard $\boldsymbol{P}^{N-1}$ as a linear subvariety of $\boldsymbol{P}^{N}$ and let $S^{*}$ be a cone in $\boldsymbol{P}^{N}$ projecting $S^{\prime}$ from a point $v$ not in $\boldsymbol{P}^{N-1}$. Then there is a quadric hypersurface $W$ in $\boldsymbol{P}^{N}$ such that $Z$ is isomorphic to $W \cdot S^{*}$.

(b) In the other direction, let $S$ be a nonsingular surface embedded in $\boldsymbol{P}^{N-1}$ so that it is projectively normal. Regard $\boldsymbol{P}^{N-1}$ as a linear subvariety of $\boldsymbol{P}^{N}$ and let $S^{*}$ be a cone in $\boldsymbol{P}^{N}$ projecting $S$ from a point $v$ not in $\boldsymbol{P}^{N-1}$; $S^{*}$ is normal. Let $W$ be a quadric hypersurface in $\boldsymbol{P}^{N}$ not passing through $v$; then $W$ and $S^{*}$ intersect properly in $\boldsymbol{P}^{N}$; suppose that $Z=W \cdot S^{*}$ is nonsingular. The projection from $v$ induces a finite flat morphism $\pi: Z$ $\rightarrow S$ of degree 2; and when $B$ is the branch curve on $S$ we have that $B$ $\sim 2 D$, where $D$ is a section of $S$ by a hyperplane in $\boldsymbol{P}^{N-1}$.

Proof. (a) By assumption there is a form $G^{\prime}$ of degree 2 in $\boldsymbol{P}^{N-1}$ such that the homogeneous equation $G^{\prime}=0$ defines the curve $B^{\prime}=\varphi(B)$ on the embedded surface $S^{\prime}$ in $\boldsymbol{P}^{N-1}$. Assume $v=(0: \cdots: 0: 1)$, say $\boldsymbol{P}^{N-1}$ is defined in $\boldsymbol{P}^{N}$ by the homogeneous equation $X_{N}=0$, let $G=X_{N}^{2}-G^{\prime}$, and take $W$ to be the quadric defined by $G=0 . \quad v$ is not on $W$. Let $Z^{\prime}=W \cdot S^{*}$ and let $\pi^{\prime}: Z^{\prime} \rightarrow S^{\prime}$ be the morphism induced by projection from $v . \quad \pi^{\prime}$ is finite ([17], p. 246) and flat (ibid. p. 432) and clearly branches over $B^{\prime}$. So, identifying $S^{\prime}$ and $B^{\prime}$ with $S$ and $B$, we have that $\pi: Z$ $\rightarrow S$ and $\pi^{\prime}: Z^{\prime} \rightarrow S^{\prime}$ are both finite double covers of $S$ with branch locus $B$; but there is just one of those up to isomorphisms (0.1).

Remark that the pointless cone $S^{*}-v$ is canonically identified ${ }^{*}$ with the line bundle $\mathcal{O}_{S}(1)=[D]$ so under the hypotheses of (a) the general

(*) The same transition functions serve to identify, both fibre coordinates in the bundle, as well as generator coordinates in the cone with value $\infty$ at $v$. 
double-cover construction of (0.1) is realized in one projective space. For this to be possible it is at very least necessary that $B$ be connected, as part (b) shows.

(b) We have to show that $B \sim 2 D$. Let $p: S^{*} \rightarrow S$ be the projection from the vertex; $\pi$ is the restriction of $p$ to $Z$ and is a proper morphism. Let $K_{S}$ be a canonical divisor on $S$ and let $H$ be a section of $S^{*}$ by a hyperplane in $\boldsymbol{P}^{N}$. A canonical divisor on $S^{*}$ is found directly to be $p^{-1}\left(K_{S}\right)-H$, so by adjunction on the normal cone $S^{*}$ we have that $K_{Z}$ $=Z \cdot\left(p^{-1}\left(K_{S}\right)+H\right)$ is a canonical divisor on $Z$, using that $Z=W \cdot S^{*} \sim$ 2H. Now we can take for $H$ a divisor $p^{-1}\left(H_{S}\right), H_{S}$ a hyperplane section of $S$ in $P^{N-1}$. Then $K_{Z}=Z \cdot\left(p^{-1}\left(K_{S}+H_{S}\right)\right)$. Let $j: Z \rightarrow S^{*}$ be the injection mapping and let $q$ be the restriction of $p$ to $S^{*}-v$; then $\pi=q \circ j$ and $\pi^{-1}\left(K_{S}+H_{S}\right)=j^{-1}\left(q^{-1}\left(K_{S}+H_{S}\right)\right)$, [26] p. 238. Furthermore, the latter cycle $=Z \cdot\left(q^{-1}\left(K_{S}+H_{S}\right)\right)=Z \cdot\left(p^{-1}\left(K_{S}+H_{S}\right)\right)$. Thus $K_{Z}=\pi^{-1}\left(K_{S}+H_{S}\right)$ so $\pi\left(K_{Z}\right)$ $=2 K_{S}+2 H_{S} \sim 2 K_{S}+2 D$. But the branch curve $B$ is linearly equivalent to $\pi\left(K_{Z}\right)-2 K_{S}$ (the characteristic $\neq 2$ ) so $B \sim 2 D$.

Let a $K 3$ surface $X$ and map $\pi: X \rightarrow F_{0}$ branched over $B$ be given as above. The complete linear system $\Lambda(24)$ on $F_{0}$ defines a nondegenerate embedding of $F_{0}$ into $P^{8}$; call $S$ this model of $F_{0} . \quad S$ is projectively normal in $\boldsymbol{P}^{8}$. Regard $\boldsymbol{P}^{8}$ as a linear subvariety of $\boldsymbol{P}^{9}$ and let $S^{*}$ be a cone in $\mathbf{P}^{9}$ projecting $S$ from a point not in $\boldsymbol{P}^{8}$. Our proposition shows that there is a quadric hypersurface $W$ in $\boldsymbol{P}^{9}$ such that $X$ is isomorphic to $W \cdot S^{*}$. We observe that $S$ and $S^{*}$ can be taken to be rational over the prime field. Note also that $W$ does not contain $S$.

This is the desired projective model of $X$. It was essentially displayed in [22] p. 253.

Next we propose to deform $S^{*}$ to a projective model of $\boldsymbol{P}^{3}$, using the following intuitively simple procedure (cf. proof of Bezout's theorem in [17]). Here $\left(Y_{0}, \cdots, Y_{m}\right)$ denote homogeneous coordinates in $\boldsymbol{P}^{m}$ and $H_{i}$ is the hyperplane defined by setting $Y_{i}=0 . \quad k$ is an algebraically closed field of definition for the variety $V . A$ is the affine line.

Proposition 2. Let $V^{n}$ be a nonsingular variety embedded in $\boldsymbol{P}^{m}$. Fix $r<n$. Assume that $V$ and the $r$ hyperplanes $H_{m-r+1}, \cdots, H_{m}$ intersect properly in $\boldsymbol{P}^{m}$ and that every component of $F^{n-r}=\left(V \cdot H_{m-r+1}, \cdots, H_{m}\right)$ has multiplicity one. Let $F^{*}$ denote the $n$-dimensional cone projecting $F$ from the center $L^{r-1}$ defined by setting $Y_{0}=\cdots=Y_{m-r}=0$. Let $t$ be a variable quantity over $k$ and regard $t$ as a generic point of $A$ over $k$. 
Then there exists a linear automorphism $T_{t}$ of $\boldsymbol{P}^{m}$, rational over $k(t)$, and a variety $\mathscr{V}^{n+1}$ on $\boldsymbol{P}^{m} \times A$, rational over $k$, such that when we set $V_{t}=T_{t}(V)$, then

$$
\begin{aligned}
V_{t} \times t & =\mathscr{V} \cdot\left(\boldsymbol{P}^{m} \times t\right), \\
F^{*} \times 0 & =\mathscr{V} \cdot\left(\boldsymbol{P}^{m} \times 0\right) .
\end{aligned}
$$

Proof. Define $T_{t}$ by setting $Z_{i}=Y_{i}$ for $0 \leqslant i \leqslant m-r, Z_{j}=(1 / t) Y_{j}$ for $m-r+1 \leqslant j \leqslant m$.

Let $M$ be a generic point of $V_{t}$ over $k(t)$ and define $\mathscr{V}^{n+1}$ to be the locus of $M \times t$ over the algebraically closed field $k$, on $\boldsymbol{P}^{m} \times A$. Then $\mathscr{V} \cdot\left(\boldsymbol{P}^{m} \times t\right)=V_{t} \times t$ by Th. 6, Ch. 8 in [26].

Let $\left\{F_{\mu}(Y)=0\right\}$ be a set of homogeneous equations for $V / k$. Then $\left\{F_{\mu}\left(Y_{0}, \cdots, Y_{m-r}, t Y_{m-r+1}, \cdots, t Y_{m}\right)=0\right\}$ is a set of homogeneous equations for $V_{t} / k(t)$. Let $G_{\mu}(Y ; T)=F_{\mu}\left(Y_{0}, \cdots, Y_{m-r}, T Y_{m-r+1}, \cdots, T Y_{m}\right)$ ( $T$ is an indeterminate). We have that the zeros-set $\mathscr{Z}\left(\left\{G_{\mu}\right\}\right) \supset \mathscr{V}$ since $G_{\mu}(M ; t)$ $=0$ and the $G_{\mu}$ are $k$-rational polynomials. Therefore $\mathscr{Z}\left(\left\{G_{\mu}\right\}, T\right) \supset \mathscr{V} \cap$ $\boldsymbol{P}^{m} \times 0$. Since $\boldsymbol{P}^{m}$ is complete, $\mathscr{V}$ is complete over every point of $A$ so $\mathscr{V} \cap \boldsymbol{P}^{m} \times 0$ is nonempty; then any component has dimension at least $n$. Now $\left\{F_{\mu}\left(Y_{0}, \cdots, Y_{m-r}, 0, \cdots, 0\right)=0\right\}$ is a set of equations for the cone $F^{*}$ in $\boldsymbol{P}^{m}$. Then certainly $\mathscr{Z}\left(\left\{G_{\mu}\right\}, T\right) \subset F^{*} \times 0$. By the hypotheses, $F^{*}$ is a purely $n$-dimensional cycle. Therefore each component of $\mathscr{V} \cap \boldsymbol{P}^{m} \times 0$ is a component of $F^{*} \times 0$.

Now let $F^{* \prime}$ be a component of the cone $F^{*} ; F^{* \prime}$ is a cone with center $L . \quad F^{*}$ projects $F$ from $L$ which is disjoint from the linear span of the base $F$; then projection from $L$ defines an isomorphism of $F$ (i.e. $F$ and $F^{*}$ have essentially the same defining equations); consequently $F^{\prime}=F^{* \prime} \cap F$ is the uniquely determined component of $F$ such that $F^{* \prime}$ is the cone $F^{* *}$ projecting $F^{\prime}$ from $L$. Hence, as $F$ is defined over the algebraically closed field $k$, there is a point $u^{\prime}$ in $F^{\prime}$ which is $k$-rational and lies on no component of $F^{*}$ except $F^{* \prime}$.

$F$ is left pointwise fixed by $T_{t}$ so $F^{\prime}$ lies on $V_{t}$. Then $u^{\prime}$ is a specialization of $M$ over $k(t)$, hence $u^{\prime} \times t$ is a specialization of $M \times t$ over $k$. Therefore $u^{\prime} \times t \in \mathscr{V}$. We'll show that $u^{\prime} \times 0 \in \mathscr{V}$. Suppose $u^{\prime}$ has a representative on the affine piece of $\mathscr{V}$ where $Y_{j} \neq 0$-call it $u_{j}^{\prime}$. Assume that $h\left(u_{j}^{\prime}, t\right)=0, h$ being a polynomial with coefficients in $k$. As a polynomial in $t$ with coefficients in $k\left[u^{\prime}\right]=k, h$ vanishes identically since $t$ is variable over $k$. Then $h\left(u_{j}^{\prime}, 0\right)=0$ too; hence $u^{\prime} \times t \rightarrow u^{\prime} \times 0$ ref. $k$, 
so $u^{\prime} \times 0 \in \mathscr{V}$. Hence $u^{\prime} \times 0 \in \mathscr{V} \cap \boldsymbol{P}^{m} \times 0$. By choice of $u^{\prime}$ and the result in the previous paragraph but one, the component of $\mathscr{V} \cap \boldsymbol{P}^{m} \times 0$ containing $u^{\prime} \times 0$ must be $F^{* \prime} \times 0$. Hence $F^{* \prime} \times 0$ is a component of $\mathscr{V} \cap \boldsymbol{P}^{m} \times 0$.

Thus $\mathscr{V} \cdot\left(\boldsymbol{P}^{m} \times 0\right)$ and $F^{*} \times 0$ have at least the same components. To show that the coefficient of a component $F^{* \prime} \times 0$ is unity in $\mathscr{V} \cdot\left(\boldsymbol{P}^{m}\right.$ $\times 0$ ), we may show that $\mathscr{V}$ and $\boldsymbol{P}^{m} \times 0$ are transversal at some point of $F^{* \prime} \times 0$ on $\boldsymbol{P}^{m} \times A$. We compute the rank of the Jacobian matrix for the equations induced by $\left\{T=0, G_{\mu}=0\right\}$ on a convenient affine piece of $\boldsymbol{P}^{m} \times A$ : the rank should be $m-n+1$. By hypothesis there is some point $z$ of $F^{\prime}$ where, on a suitable affine piece of $\boldsymbol{P}^{m}$, the rank of the Jacobian matrix for the equations induced by $\left\{F_{\mu}=Y_{m-r+1}=\cdots=Y_{m}=0\right\}$ is $m-n+r$. By rearranging $[0,1, \cdots, m-r]$ as needed we may take the affine piece where $Y_{0} \neq 0$ (since $Y_{m-r+1}=\cdots=Y_{m}=0$ ). Set $y_{i}=Y_{i} / Y_{0}$. Then, the rank $\left\|\partial F_{\mu} / \partial y_{i}\right\|, 1 \leqslant i \leqslant m-r$, is $m-n$ at $z$. Consequently, rank $\left\|\partial G_{\mu} / \partial y_{i}\right\|, 1 \leqslant i \leqslant m-r$, is $m-n$ at $(z, 0)$ since $\partial G_{\mu} / \partial y_{i}(z, 0)=\partial F_{\mu} / \partial y_{i}(z)$ for such $i$. Then the rank of the matrix for the equations induced by $\left\{T=0, G_{\mu}=0\right\}$ is $m-n+1$ at $(z, 0) \in F^{* \prime} \times 0$. This ends the proof.

CoROLLARY. Let $k_{0}$ be the algebraic closure of the prime field. Let $S / k_{0}$ be a nonsingular surface in $\boldsymbol{P}^{8}$, which is the reembedding of a quadric surface $S_{0}$ in $\boldsymbol{P}^{3}$ by the linear system of quadrics in $\boldsymbol{P}^{3}$. Regard $\boldsymbol{P}^{8}$ as a linear subvariety of $\boldsymbol{P}^{9}$ and let $S^{*}$ be the cone projecting $S$ from a point $v$ not in $P^{8}$. Let $t$ be as in the Proposition.

Then there is a variety $V_{t}$ in $\boldsymbol{P}^{9}$, isomorphic to $\boldsymbol{P}^{3}$ over $k_{0}(t)$, and $a$ variety $\mathscr{V}^{4}$ in $\boldsymbol{P}^{9} \times A$, rational over $k_{0}$, such that $V_{t} \times t=\mathscr{V} \cdot\left(\boldsymbol{P}^{9} \times t\right)$, $S^{*} \times 0=\mathscr{V} \cdot\left(\boldsymbol{P}^{9} \times 0\right)$.

Proof. We may assume that $S$ is contained in the hyperplane $H$ defined by $Y_{9}=0$ and that $v=(0: \cdots: 0: 1)$. In the vector space of forms of degree 2 in $P^{3}$, choose a basis of forms $\sigma_{0}, \cdots, \sigma_{9}$ rational over $k_{0}$ so that $S_{0}$ is defined by $\sigma_{9}=0$ in $\boldsymbol{P}^{3}$. Let $V / k_{0}$ be the image of $\boldsymbol{P}^{3}$ when it is embedded into $P^{9}$ by the map $Y_{i}=\sigma_{i}, 0 \leqslant i \leqslant 9$, and change $\sigma_{0}, \cdots, \sigma_{8}$ if necessary so that $S$ is the image of $S_{0}$ by the same map. Then $V \cdot H$ $=S$.

Define $T_{t}$ by $T_{t}\left(Y_{0}, \cdots, Y_{9}\right)=\left(Y_{0}, \cdots, Y_{8},(1 / t) Y_{9}\right)$ and put $V_{t}=T_{t}(V)$. Then $V_{t}$ is isomorphic to $\boldsymbol{P}^{3}$ over $k_{0}(t)$. The Corollary follows when we put $m=9, n=3, r=1$ in the Proposition. 
Now let $S / k_{0}$ be as in the Corollary. We have seen that there is a quadric hypersurface $W$ in $\boldsymbol{P}^{9}$ such that our $K 3$ surface $X$ is isomorphic to $W \cdot S^{*}$. Let $k=\bar{k}=\operatorname{def}(X, W, S)$, let $t$ be variable over $k$, and let $V_{t}$ be as in the Corollary. The Corollary shows that in fact $S^{*}$ is a specialization of $V_{t}$ over $k$, as cycles in $\boldsymbol{P}^{9} / k$, at least in the sense of cycles on product varieties, [26]. $W$ is rational over $k$, so $\left(S^{*}, W\right)$ is a specialization of $\left(V_{t}, W\right)$ over $k$. Then $S^{*} \cdot W$ is a specialization of $V_{t} \cdot W$ over $k$, [23] p. 104. Since the former is irreducible and nonsingular, the latter is also. As $V_{t} \cdot W=V \cdot T_{t}^{-1}(W)$ over $k(t), V_{t} \cdot W$ is isomorphic to a quartic surface in $\boldsymbol{P}^{3}$, by definition of $V$ as a reembedding of $\boldsymbol{P}^{3}$ by quadrics. Setting $\boldsymbol{Q}$ $=V_{t} \cdot W$, we have shown all but the final sentence of the following.

TheOREM 1. Let a K3 surface $X / k$ be given, as above, and let $t$ be variable over $k$. There exists a surface $Q / k(t)$, isomorphic to a nonsingular quartic surface in $\boldsymbol{P}^{3}$, such that $X$ is a specialization of $Q$ over $k$.

Moreover, take for basic polar curves (in the sense of [13]) on the surfaces a nonsingular non-hyperelliptic curve $C$ of genus 3 on $Q$, and $a$ nonsingular hyperelliptic curve $D$ of genus 3 on $X$. Then the specialization $Q \rightarrow X$ is a specialization of polarized surfaces.

Proof. Let $U^{2} \subset V$ be the image of a plane in $\boldsymbol{P}^{3}$ by the isomorphism $\boldsymbol{P}^{3} \widetilde{I}$, and set $U_{t}=T_{t}(U) . \quad U \cdot H=U_{t} \cdot H$ is a twisted quartic curve $R$ (= nonsingular rational curve spanning a $P^{4}$ ). We may assume that $U_{t} \cdot{ }_{V} Q Q$ is isomorphic to a nonsingular quartic curve in $\boldsymbol{P}^{2}$, of genus 3 . In the course of the specialization of $V_{t}$ to $S^{*}, U_{t}$ will specialize to a 2-dimensional cone $R^{*}$ projecting $R$ from $v$, by Prop. $2\left(U_{t}\right.$ is a Veronese surface). Now $Q \cdot{ }_{V_{t}} U_{t}=\left(W \cdot{ }_{P^{9}} V_{t}\right) \cdot{ }_{V_{t}} U_{t}=W \cdot{ }_{P^{9}} U_{t}$ and similarly $X \cdot{ }_{S^{*}} R^{*}$ $=\left(W \cdot{ }_{p_{9}} S^{*}\right) \cdot{ }_{S^{*}} R^{*}=W \cdot{ }_{p_{9}} R^{*}$. Then, by compatibility of specialization with intersection product, we may take $Q \cdot{ }_{V_{t}} U_{t}$ for $C$, and $X \cdot{ }_{S^{*}} R^{*}$ for $D$.

\section{§2. Algebraic elementary operations}

2.1. A curve $R$ on a surface $S$ is called a nodal curve if $S$ is nonsingular at all points of $R$ and $R$ is an irreducible nonsingular rational curve with self-intersection -2 (terminology of [3]).

Let $C$ be a nonsingular curve, let $\mathscr{S}^{3}$ be a variety and let $p: \mathscr{S} \rightarrow \mathrm{C}$ be a proper morphism defining a family of nonsingular surfaces. Let 0 be a point of $C$, rational over a field of definition for $p$, and suppose that $S=p^{-1}(0)$ carries a nodal curve $R$ with does not extend locally in $\mathscr{S}$, 
that is a relative divisor in some neighborhood $U$ of $R$ in $\mathscr{S}$. An algebraic elementary operation in $\mathscr{S}$ along $R$ is a birational transformation $\eta: \mathscr{S} \rightarrow \mathscr{S}^{*}$ such that $\mathscr{S}^{*}$ carries a nonsingular rational curve $R^{*}, \eta$ induces a biregular isomorphism between $\mathscr{S}-R$ and $\mathscr{S}^{*}-R^{*}$, and the total $\eta$-transform of any point of $R$ is the whole curve $R^{*}$. Let $p^{*}=p \circ \eta^{-1}$. We have that $p^{*-1}(0)=\eta \circ p^{-1}(0)=\eta S\left([26]\right.$ p. 238); call $S^{*}$ the surface $p^{*-1}(0)$. We shall assume as part of the definition that $S^{*}$ is normal. Let $f$ be the mapping $f: S \rightarrow S^{*}$ induced by $\eta$. Clearly $R^{*}$ lies on $S^{*}$ and $f$ is birational and biregular between $S-R$ and $S^{*}-R^{*}$. Moreover $f$ is defined at $R$ and at $R^{*}$. Let us see that $f$ is biregular at all points.

It is enough to show that, if $f$ is not everywhere biregular, $R$ must be exceptional for $f$, i.e. the geometric image of $R$ must be one point of $R^{*}$, since in fact $R^{2}<-1$ precludes that $R$ be exceptional, [29] p. 76. For this it suffices to show that if either $f$ or $f^{-1}$ is everywhere defined, then so is the other map. For then if $f$ is not everywhere biregular, $f^{-1}$ is not everywhere defined; hence $f^{-1}$ is not defined at some closed point $P^{*}$ of $R^{*}$; hence there is a component $D$ of $\Gamma_{f} \cap\left(S \times P^{*}\right)$ of dimension 1 , on account of the normality of $S^{*}$ (Zariski's main theorem, [27]; cf. [26] pp. 200,313$)$. $D$ clearly projects onto $R$ so $f(R)=P^{*}$ since $f$ is defined at $R$, and $R$ is exceptional for $f$.

So suppose $f$ is a morphism. Let $k$ be a field of definition for $f, R$ and $R^{*}$, and let $P^{*}$ be a generic point of $R^{*}$ over $k . f^{-1}\left(P^{*}\right)$ cannot be a closed point of $R$ since $f$ is a morphism, hence $f^{-1}\left(P^{*}\right)$ is a generic point $P$ of $R$ over $k$. Then $f(P)=P^{*}$ so $f^{-1}\left(P^{+}\right) \neq P$ for any $k$-closed point $P^{+}$of $R^{*}$. $f^{-1}$ must then be finite-valued at every point of $R^{*}$, hence a morphism by Zariski's main theorem again.

Thus $f$ is an isomorphism and $S^{*}$ is nonsingular, if $S^{*}$ is normal. ( $R$ could be called "a fundamental curve of 2 nd kind", [27] p. 516, in $\mathscr{S}$.) The graph of $\eta$ contains $R \times R^{*}$, and $R^{*}$ is a nodal curve on $S^{*}$, so algebraic elementary operations are reflexive operations.

We shall try to perform algebraic elementary operations following the procedure of Horikawa in [9], which we now recall.

Let $\mathscr{N}$ be the sheaf of germs of sections of the normal bundle of $R$ in $\mathscr{S} . \mathscr{N}$ is a sheaf extension of $\mathcal{O}_{R}(-2)$ by $\mathcal{O}_{R}$, i.e. there is an exact sequence $0 \rightarrow \mathcal{O}_{R}(-2) \rightarrow \mathscr{N} \rightarrow \mathcal{O}_{R} \rightarrow 0$ of sheaves on $R$; the group of such extensions is $\operatorname{Ext}_{\mathscr{O}_{R}}^{1}\left(\mathcal{O}_{R}, \mathcal{O}_{R}(-2)\right) \cong H^{1}\left(R, \mathcal{O}_{R}(-2)\right)$ which is one-dimensional since $R \cong \boldsymbol{P}^{1}$. Hence there is just one nontrivial class of extensions; one 
sees directly (cf. (0.1)) that $\mathcal{O}_{R}(-1) \oplus \mathcal{O}_{R}(-1)$ is a nontrivial extension so there are two cases: either (a) $\mathscr{N}=\mathcal{O}_{R}(-2) \oplus \mathcal{O}_{R}$, or (b) $\mathscr{N}=\mathcal{O}_{R}(-1)$ $\oplus \mathcal{O}_{R}(-1)$. Let us blow up along $R$ in $\mathscr{S}$. Let $\pi: \mathscr{S}_{1} \rightarrow \mathscr{S}$ be the monoidal transformation. The exceptional surface $E=\boldsymbol{P}(\mathscr{N})$ is isomorphic (0.1) to the ruled surface $F_{2}$ in case (a), or to $F_{0}$ in case (b). The sub-invertiblesheaf $\mathcal{O}_{R}(-2) \subset \mathscr{N}$ corresponds to $\Delta$ on $\boldsymbol{P}(\mathscr{N})$, therefore the proper transform of $S$ intersects $E$ in $\Delta$, on $\mathscr{S}_{1}$. Now $\Delta$ and $b$ are disjoint in case (a), hence in that case $R_{1}=b$ is a nodal curve on the (reducible) member $p_{1}^{-1}(0)$ of the family $p_{1}: \mathscr{S}_{1} \rightarrow C$, where $p_{1}=p \circ \pi$. We may proceed to analyse the normal bundle of $R_{1}$ in $\mathscr{S}_{1}$ as before. If case (a) occurs again, so does another nodal curve $R_{2}$ lying on $E_{2}$ in $\mathscr{S}_{2}$ after blowing up-and so on.

Consider the relation of the existence of this succession of nodal curves $R_{n}$ to the question whether $R$ extends locally in $\mathscr{S}$. It is easy to see that, if $R$ extends locally then case (a) always occurs at each blowing up. In the analytic case, Horikawa shows that the converse is true: if $R$ does not extend then case (b) must occur sooner or later. Indeed, in the analytic case $\mathscr{S}$ is analytically isomorphic along $R$ to a reparametrization of the "standard example" of $F_{0}$ degenerating to $F_{2}$, and the number of monoidal transformations required to reach case (b) has a nice relation to the reparametrization, [9].

But in the algebraic case we must, at least at present, simply assume that case (b) occurs eventually when $R$ does not extend locally in $\mathscr{S}$. (In the Appendix (A.1) a formal analogue of Horikawa's result is proved.) So assume that after finitely many successive monoidal transformations $\mathscr{S} \leftarrow \mathscr{S}_{1} \leftarrow \cdots \leftarrow \mathscr{S}_{n}$ along nodal curves $R, R_{1}, R_{2}, \cdots, R_{n-1}$ we have a variety $\mathscr{S}_{n}$ containing a "scaffolding" of transforms of exceptional surfaces $E_{1}, E_{2}, \cdots, E_{n-1}$, all isomorphic to $F_{2}$, and "on top" an exceptional surface $E=E_{n} \cong F_{0}$. Let $\langle\ell\rangle$ be the ruling on $E$ (cf. (0.1)) consisting of blown-up points of $R_{n-1}$ and let $\left\langle\ell^{\prime}\right\rangle$ be the other ruling, and let $\rho: E \rightarrow P^{1}$ be the natural projection of $E$ which collapses members of $\left\langle\ell^{\prime}\right\rangle$ to points of $\boldsymbol{P}^{1}$. We try to find a sequence of morphisms $\mathscr{S}_{n} \rightarrow \mathscr{S}_{n-1}^{*} \rightarrow \cdots \rightarrow \mathscr{S}^{*}$ such that $\mathscr{S}_{n} \rightarrow \mathscr{S}_{n-1}^{*}$ induces $\rho$ on $E$ and $\mathscr{S}_{n-1}^{*} \rightarrow \cdots \rightarrow \mathscr{S}^{*}$ "dismantles the scaffolding," that is blows down the transforms of $E_{n-1}, \cdots, E_{1}$. When such morphisms exist, let $\pi^{*}: \mathscr{S}_{n} \rightarrow \mathscr{S}^{*}$ be their composition and let $\pi: \mathscr{S}_{n} \rightarrow \mathscr{S}$ be the composition of the first set of transformations. Then $\eta=\pi^{*} \circ \pi^{-1}$ is an algebraic elementary operation in $\mathscr{S}$ along $R$. 
In what follows we shall use the results of Lascu [12] to find some sufficient conditions for $\pi^{*}$ and hence $\eta$ to exist in the algebraic case.

2.2. Let $X$ and $Y$ be distinct irreducible surfaces embedded in a nonsingular 3-fold $V$. Let $C$ be an irreducible nonsingular curve in $V$, let $\pi: V^{\prime} \rightarrow V$ be the monoidal transformation in $V$ along $C$ and let $E=\pi^{-1}(C)$ be the exceptional surface in $V^{\prime} . X$ and $Y$, being divisors, intersect properly in $V$; assume that $C$ is a component of $X \cdot Y$ with multiplicity one, and that every point of $C$ is simple on $X$. Write $X \cdot Y=C+D$. Call $X^{\prime}, Y^{\prime}$ the proper transforms of $X, Y$ in $V^{\prime}$; these intersect properly with the divisor $E$; let $A=X^{\prime} \cdot E, B=Y^{\prime} \cdot E$.

Proposition 3. Assumptions and notations being as above, the following assertions are equivalent: (a) $D$ intersects $C$ at a single point $P$, simple on $D$ ( $C$ is nonsingular), and $C$ and $D$ have distinct tangents at $P$; (b) $A$ and $B$ intersect with multiplicity one on $E$.

Proof. Call $D^{\prime}$ the proper transform of $D=X \cdot Y-C$. Let us see first that $D^{\prime}=X^{\prime} \cdot Y^{\prime}$. Indeed, $\pi$ induces an isomorphism between $V^{\prime}-E$ and $V-C$, so $0 \preccurlyeq X^{\prime} \cdot Y^{\prime}-D^{\prime} \subset E$; suppose $D^{*}$ is an irreducible component of $X^{\prime} \cdot Y^{\prime}-D^{\prime} . \quad X$ and $Y$ have distinct tangent planes at every point of $C$ except points of $D \cap C$ (criterion of multiplicity one, [26] p. 152), so $\pi\left(D^{*}\right)$ is supported by $D \cap C$. But $\pi$ induces an isomorphism on $X^{\prime}$ since $X$ is nonsingular at all points of $C$, so in particular $\pi$ cannot collapse any curve $D^{*}$ on $X^{\prime}$ to a point; hence $D^{*}=0$, so $D^{\prime}=X^{\prime} \cdot Y^{\prime}$.

Now it is clear that $D$ intersects $C$ at a single point, simple on $D$, with distinct tangents if, and only if, $D^{\prime}$ and $E$ intersect with multiplicity one on $V^{\prime}$. On the other hand, $A \cdot{ }_{E} B=\left(X^{\prime} \cdot E\right) \cdot{ }_{E}\left(Y^{\prime} \cdot E\right)=X^{\prime} \cdot\left(Y^{\prime} \cdot E\right)$ $=\left(X^{\prime} \cdot Y^{\prime}\right) \cdot E$, so $A \cdot{ }_{E} B=D^{\prime} \cdot E$; the proof is thereby finished.

Before deriving 2 corollaries we fix some notation for the remainder of $\S 2$. Let $p: \mathscr{S} \rightarrow C$ be a proper morphism defining an algebraic family of nonsingular surfaces, as at the beginning of $\S 2$, and suppose that $S=p^{-1}(0)$ carries a nonextending nodal curve $R$. Let $\pi: \mathscr{S}^{\prime} \rightarrow \mathscr{S}$ be the monoidal transformation in $\mathscr{S}$ along $R$ and let $E=\pi^{-1}(R)$ be the exceptional surface. $E$ is isomorphic either to $F_{0}$ or to $F_{2}$, (2.1). Call $S^{\prime}$ the proper transform of $S$ in $\mathscr{S}^{\prime}$ and set $p^{\prime}=p \circ \pi$; then $p^{\prime-1}(0)=S^{\prime}+E$.

Now suppose that $E \cong F_{0}$. Let $\langle\ell\rangle$ be the ruling on $E$ consisting of blown up points of $R$, let $\left\langle\ell^{\prime}\right\rangle$ be the other ruling, and let $\Delta=S^{\prime} \cdot E$ be the diagonal on $E$. 
CoRollary 1. Let $T$ be an irreducible surface in $\mathscr{S}$ and let $T^{\prime}$ be its proper transform in $\mathscr{S}^{\prime}$. Assume $T$ intersects properly with $S$ and passes through $R$. Then $T \cdot S=R+D$ with $R$ and $D$ intersecting with multiplicity one on $S$ if, and only if, $T^{\prime} \cdot E$ is a member of the ruling $\left\langle\ell^{\prime}\right\rangle$.

Proof. By the Proposition (taking $X=S$ ), $T \cdot S=R+D$ as stated if, and only if, $T$ and $S$ intersect transversally along $R$ and $T^{\prime} \cdot E$ intersects $S^{\prime} \cdot E$ with multiplicity one on $E$. On the other hand, let $B=T^{\prime} \cdot E$; $B \geqslant 0$ so $B$ is a member of $\left\langle\ell^{\prime}\right\rangle$ if, and only if, the equations $I(B, \ell)$ $=I(B, \Delta)=1$ hold on $E$ for some $\ell$ in $\langle\ell\rangle$. Now suppose $B \in\left\langle\ell^{\prime}\right\rangle$. Then $T^{\prime} \cdot E=B$ intersects $S^{\prime} \cdot E=\Delta$ with multiplicity one on $E$. Moreover there is $\ell \in\langle\ell\rangle$ such that $B$ and $\Delta$ meet $\ell$ in different points. Let $P$ be the point of $R$ corresponding to $\ell$. As $B \cdot{ }_{E} \ell=\left(T^{\prime} \cdot E\right) \cdot{ }_{E} \ell=T^{\prime} \cdot \ell, I(B, \ell)$ $=1$ implies that $P$ and hence $R$ is simple on $T$, in fact that $\pi$ induces on $T^{\prime}$ a mapping which is biregular at $P^{\prime}=T^{\prime} \cdot \ell$, by [26] p. 258; and, as $B$ and $\Delta$ meet $\ell$ in different points, $T$ and $S$ have distinct tangent planes at $P$. It follows that $T \cdot S=R+D$ as stated.

To finish the proof in the other direction we need only observe that, if $T$ and $S$ intersect transversally along $R$ then $I(B, \ell)=1$ for at least one $\ell \in\langle\ell\rangle$.

We remark that, if the equivalent conclusions of the Corollary hold, the proof shows that $\pi$ induces on $T^{\prime}$ an isomorphism to $T$, hence that $T$ is nonsingular at all points of $R$.

Corollary 2. The Corollary 1 holds also when the condition $E \cong F_{0}$ is replaced by $E \cong F_{2}$ and the ruling $\left\langle\ell^{\prime}\right\rangle$ is replaced by the pencil $b+\langle\ell\rangle$.

Proof. Indeed, $B=T^{\prime} \cdot E$ is a member of $b+\langle\ell\rangle$ if, and only if, the equations $I(B, \ell)=I(B, \Delta)=1$ hold for some $\ell \in\langle\ell\rangle$. Then the proof of Corollary 1 works here too.

Under the assumptions and notations of Corollary 1, there is a welldefined correspondence between the members of the two rulings $\langle\ell\rangle$ and $\left\langle\ell^{\prime}\right\rangle$. Namely, for each line in one ruling there is just one line in the other ruling such that the two lines and the diagonal $\Delta=S^{\prime} \cdot E$ meet all at the same point. Thus, if $P \in R$ and $\ell_{P} \in\langle\ell\rangle$ is the corresponding member of $\langle\ell\rangle$, we may refer to $\ell_{p}^{\prime}$ in $\left\langle\ell^{\prime}\right\rangle$ : it is determined by $\ell_{P} \cdot \Delta=\ell_{p}^{\prime} \cdot \Delta$. The proof of Corollary 1 now shows that, if $P \in R$ and $T_{P}$ is an irreducible surface in $\mathscr{S}$ such that $T_{P} \cdot S=R+D$ with $R \cdot D=P$, then

$$
T_{P}^{\prime} \cdot E=\ell_{P}^{\prime}
$$


2.3. We wish to state conditions, in terms of surfaces in $\mathscr{S}$, which will imply that elementary operation in $\mathscr{S}$ along $R$ is algebraic. In the first place we recall the conditions of Lascu in our situation (Lascu [12], Lemma 5 and Remark, p. 691). We have $p^{\prime}: \mathscr{S}^{\prime} \rightarrow C$, where $p^{\prime-1}(0)=S^{\prime}+E$ with $E$ isomorphic either to $F_{0}$ or to $F_{2}$, and $S^{\prime} \cdot E=\Delta$ on $E$. Let $k$ be an algebraically closed field of definition for $p^{\prime}$. Let $\mathscr{I}$ be the sheaf on $\mathscr{S}^{\prime}$ of ideals of the divisor $E$ and let $\mathcal{O}(1)=\mathcal{O}_{E}(\Delta)$ on $E$. In $\mathscr{S}^{\prime}$ we have that $S^{\prime}+E$ is a fibre of the morphism $p^{\prime}$, hence the sequence $0 \rightarrow \mathscr{I}^{2} \rightarrow$ $\mathscr{I} \rightarrow i_{*} \mathcal{O}(1) \rightarrow 0$ is exact, where $i$ is the injection mapping of $E$ into $\mathscr{S}^{\prime}$. Let $R^{*}$ be a copy of $\boldsymbol{P}^{1}$, let $\langle\ell\rangle$ be a ruling on $E$, and let $\rho: E \rightarrow R^{*}$ be the natural projection of $E$ to $R^{*}$ whose fibres are members of $\langle\ell\rangle$. Suppose that the following two conditions are satisfied for each $k$-rational point $P \in R^{*}:\left(L_{1}\right)$ for every element $\alpha \in \mathcal{O}_{R^{*}, P}$, there exists $\bar{\alpha} \in k\left(\mathscr{S}^{\prime}\right)$, regular at every point of $\ell_{P}=\rho^{-1}(P)$, such that $\bar{\alpha} \circ i=\alpha \circ \rho$ on $E$; $\left(L_{2}\right)$ there exist sections of $\mathscr{I}$ over a neighborhood of $\ell_{P}$ in $\mathscr{S}^{\prime}$ whose images in $\Gamma\left(\ell_{P}\right.$, $\left.\mathcal{O}_{\ell_{P}}(1)\right)$ generate that 2-dimensional vector space over $k$. Then there exists a proper morphism $\pi^{*}: \mathscr{S}^{\prime} \rightarrow \mathscr{S}^{*}$, inducing $\rho$ on $E$ and biregular elsewhere in $\mathscr{S}^{\prime}-E$. Conversely, if such a $\pi^{*}$ exists then $\left(L_{1}\right)$ and $\left(L_{2}\right)$ are true.

Proposition 4. Let $R$ be a nonextending nodal curve on $S$ in $\mathscr{S}$, and suppose that $\pi^{-1}(R)=E \cong F_{0}$ with rulings $\langle\ell\rangle$, $\left\langle\ell^{\prime}\right\rangle$ as described above. Assume the following. (1) There is a linear pencil $\langle L\rangle$ of surfaces $L$ in $\mathscr{S}$ such that each $L$ intersects $R$ properly with multiplicity one and there is a unique member $L_{P}$ through each point $P$ of $R$. (2) There is a linear pencil $\langle T\rangle$ of surfaces $T$ in $\mathscr{S}$ such that for each $T, T \cdot S=R+D$ with $R$ and $D$ intersecting with multiplicity one on $S$, and for each $P$ in $R$ there is a unique member $T_{P}$ with $\left(T_{P} \cdot S-R\right) \cdot R=P$. (3) Let $L \in\langle L\rangle, T \in\langle T\rangle$. There is a surface $W$ in $\mathscr{S}$ with $W \sim L+T$ and $W \cap R$ empty.

Then the proper morphism $\pi^{*}: \mathscr{S}^{\prime} \rightarrow \mathscr{S}^{*}$ inducing $\rho$ exists as above, hence the elementary operation in $\mathscr{S}$ along $R$ is algebraic.

Proof. We have to check Lascu's conditions for members of the ruling $\left\langle\ell^{\prime}\right\rangle$, which are the fibres of $\rho: E \rightarrow R^{*}$.

$\left(L_{1}\right):$ Let $P \in R^{*}$ and $\alpha \in \mathcal{O}_{R^{*}, P}$. Write $\operatorname{div}(\alpha)=\sum a_{i} P_{i}-\sum b_{j} P_{j}$, with $a_{i}$ and $b_{j}$ positive integers and $P_{i}, P_{j}$ points of $R^{*}$, and let $\ell_{i}^{\prime}=\rho^{-1}\left(P_{i}\right)$. Then $\operatorname{div}(\alpha \circ \rho)=\sum a_{i} \ell_{i}^{\prime}-\sum b_{j} \ell_{j}^{\prime}$. For each $P_{i}$ let $T_{i} \in\langle T\rangle$ be the surface such that $T_{i}^{\prime} \cdot E=\ell_{i}^{\prime}$, according to the equation (+); likewise for $P_{j}$. The proper transforms $T^{\prime}$ of the members of $\langle T\rangle$ comprise a linear pencil $\left\langle T^{\prime}\right\rangle$ in 
$\mathscr{S}^{\prime}$, because each $T$ passes simply through $R$ in $\mathscr{S}$, so there is a function $\bar{\alpha} \in k\left(\mathscr{S}^{\prime}\right)$ with $\operatorname{div}(\bar{\alpha})=\sum a_{i} T_{i}^{\prime}-\sum b_{j} T_{j}^{\prime}$. Then $\alpha \circ \rho=\bar{\alpha} \circ i$ as functions on $E$, at least up to a constant factor. The equation $(+)$ shows that $\left\langle T^{\prime}\right\rangle$ has no base point on $E$, so $\bar{\alpha}$ is regular at every point of $\ell_{P}^{\prime}=\rho^{-1}(P)$. $\left(L_{2}\right)$ : Let $P \in R^{*}$ and $\ell_{P}^{\prime}=\rho^{-1}(P) \in\left\langle\ell^{\prime}\right\rangle$. Select some $P^{+} \in R^{*}, P^{+} \neq P$, and consider the pencil $\langle Q\rangle=\langle L\rangle+T_{P^{+}}$. For each $Q \in\langle Q\rangle$, the proper transform $Q^{\prime}$ in $\mathscr{S}^{\prime}$ satisfies $Q^{\prime} \cdot E=L^{\prime} \cdot E+\left(T_{P^{+}}^{\prime}\right) \cdot E=\ell_{P}+\ell_{P+}^{\prime}$, and the total transform is $Q^{\prime}+E$ since $Q$ passes simply through $R$ in $\mathscr{S}$. Therefore the pencil $\left\langle Q^{\prime}\right\rangle$ cuts the linear series of degree 1 on the line $\ell_{P}^{\prime}$. Furthermore, when $Q \in\langle Q\rangle$ there is a linear equivalence $Q \sim W$, so there is a function $f$ in $k(\mathscr{S})$ with $\operatorname{div}(f)=Q-W$; then this $f$, taken in $k\left(\mathscr{S}^{\prime}\right)$, defines a section of $\mathscr{I}$ over a neighborhood of $E$. Any two such $f$, corresponding to distinct members of $\langle Q\rangle$, satisfy condition $\left(L_{2}\right)$.

In (A.2) we examine the case in which more than one monoidal transformation is required for case (b) to occur (cf. (2.1)).

\section{§3. Examples}

In this section we give an example of an algebraic family $\mathscr{K}$ of $K 3$ surfaces, one of whose members carries a nonextending nodal curve, such that the elementary operation in $\mathscr{K}$ along that curve is algebraic. Also we discuss some cases of similar families in which the elementary operation is not algebraic. Here $k_{0}$ denotes the algebraic closure of the prime field, characteristic $\neq 2 ; A$ is the affine line.

3.1. Proposition 5. (cf. [11], [2], [12]) (a) There is a variety $\mathscr{M}$ and a proper morphism $\varphi: \mathscr{M} \rightarrow A$, rational over $k_{0}$, defining a family of nonsingular surfaces whose members are as follows. $M_{0}=\varphi^{-1}(0)$ is isomorphic to $F_{2}$ and $M_{x}=\varphi^{-1}(x)$ is isomorphic to $F_{0}$ when $x \in A, x \neq 0$. (b) $\mathscr{M}$ carries two pencils of surfaces $\langle T\rangle$ and $\langle L\rangle$ such that $\langle T\rangle$ (resp. $\langle L\rangle$ ) cuts the ruling $\langle\ell\rangle$ (resp. the ruling $\left\langle\ell^{\prime}\right\rangle$ ) on $M_{x}, x \neq 0$, and the pencil $\langle\ell\rangle+b$ (resp. the ruling $\langle\ell\rangle$ ) on $M_{0}$, where $b$ is the nodal curve on $M_{0}$, cf. (0.1).

(c) The normal bundle of $b$ in $\mathscr{M}$ is isomorphic to $\mathcal{O}_{b}(-1) \oplus \mathcal{O}_{b}(-1)$. (d) $\mathscr{M}$ contains a surface $W$, disjoint from $b$, with $W \sim T+L$ when $T \in\langle T\rangle$, $L \in\langle L\rangle$.

Proof. Let $V^{\prime}=P^{3} \times A$, let $\left(X_{0}: X_{1}: X_{2}: X_{3}\right)$ be homogeneous coordinates in $\boldsymbol{P}^{3}$ and let $t$ be a nonhomogeneous coordinate on $A$. Define a 3-fold $\mathscr{M}^{\prime}$ in $V^{\prime}$ by the equation $X_{1}\left(X_{1}-t X_{0}\right)=X_{2} X_{3}$. $\mathscr{M}^{\prime}$ is easily seen 
to be nonsingular except at $(1: 0: 0: 0 ; 0)$ where it is normal. Let $\left\langle T^{\prime}\right\rangle$ and $\left\langle L^{\prime}\right\rangle$ be linear pencils of surfaces in $\mathscr{M}^{\prime}$ with equations

$$
T_{\lambda}^{\prime}:\left\{X_{1}-t X_{0}=\lambda X_{2}, X_{3}=\lambda X_{1}\right\} ; L_{\lambda}^{\prime}:\left\{X_{1}-t X_{0}=\lambda^{\prime} X_{3}, X_{2}=\lambda^{\prime} X_{1}\right\},
$$

where $\lambda, \lambda^{\prime}$ are nonhomogeneous parameters. The natural projection $V^{\prime}$ $\rightarrow A$ induces a morphism $\pi^{\prime}: \mathscr{M}^{\prime} \rightarrow A$ whose fibres are isomorphic to nonsingular quadric surfaces in $\boldsymbol{P}^{3}$, except $\pi^{\prime-1}(0)$ is a quadric cone in $\boldsymbol{P}^{3}$ whose vertex is $v=(1: 0: 0: 0)$. Moreover the pencils $\left\langle T^{\prime}\right\rangle$ and $\left\langle L^{\prime}\right\rangle$ in $\mathscr{M}^{\prime}$ cut the two rulings on each nonsingular quadric fibre, and each pencil cuts the one ruling on the cone (the intersection products are taken in $\left.\mathscr{M}^{\prime}\right)$. Now let $\bar{\mu}: V \rightarrow V^{\prime}$ be the monoidal transform of $V^{\prime}$ with center $T_{\infty}^{\prime}:\left\{X_{1}=X_{2}=0\right\}$, let $\mathscr{M}$ be the proper transform of $\mathscr{M}^{\prime}$ in $V$, and let $\mu: \mathscr{M}$ $\rightarrow \mathscr{M}^{\prime}$ be the induced morphism. $\mu$ is biregular everywhere except over $v$ so we consider the affine open subset $U^{\prime}$ of $\mathscr{M}^{\prime}$ where $X_{0} \neq 0$, which contains $v$. We set $\left(x_{1}, x_{2}, x_{3}, t\right)=\left(X_{1} / X_{0}, X_{2} / X_{0}, X_{3} / X_{0}, t\right)$ and set $\left(z_{1}, \zeta_{1}, t\right)$ $=\left(x_{2} / x_{1}, x_{3}, t\right),\left(z_{2}, \zeta_{2}, t\right)=\left(x_{1} / x_{2}, x_{2}, t\right)$. Let $U_{1}$ and $U_{2}$ be the affine open subsets of $\mathscr{M}$ where $z_{2} \neq 0$ and $z_{1} \neq 0$ respectively. Then $\mu^{-1}\left(U^{\prime}\right)$ is covered by $U_{1} \cup U_{2}$ and one sees easily that $\left(z_{i}, \zeta_{i}, t\right)$ are uniformizing parameters in $U_{i}, i=1,2$ and in fact that the parameters define isomorphisms from $U_{i}$ to $A^{3}$. Moreover the parameters are related by equations $z_{2}=z_{1}^{-1}$, $\zeta_{2}=z_{1}^{2} \zeta_{1}+t z_{1}$. Let $\varphi: \mathscr{M} \rightarrow A$ be the morphism defined by $\varphi=\pi^{\prime} \circ \mu$. It follows that fibres of $\varphi$ are as asserted in (a) (cf. [11]).

Next we pull back $\left\langle T^{\prime}\right\rangle$ and $\left\langle L^{\prime}\right\rangle$ via $\mu$ to get two pencils $\langle T\rangle$ and $\langle L\rangle$ whose members have local equations in $U_{1}, U_{2}$ as follows:

$$
T_{\lambda}:\left\{\zeta_{1}=\lambda\left(z_{1} \zeta_{1}+t\right), \lambda \zeta_{2}=z_{2} \zeta_{2}-t\right\} ; L_{\lambda^{\prime}}:\left\{z_{1}=\lambda^{\prime}, 1=\lambda^{\prime} z_{2}\right\} .
$$

The nodal curve $b$ on $M_{0}$ is contained in $U_{1} \cup U_{2}$ and is defined on $U_{1}$ by local equations $\zeta_{1}=0, t=0$ and on $U_{2}$ by $\zeta_{2}=0, t=0$. Then assertion (b) follows by inspection at once.

With respect to the covering $U_{1} \cup U_{2}$ of $b$ by affine open sets in $\mathscr{M}$, the transition matrix defining the normal bundle of $b$ is

$$
\left(\begin{array}{cc}
\bar{z}_{1}^{2} & \bar{z}_{1} \\
0 & 1
\end{array}\right),
$$

taking $\left(\zeta_{i}, t\right)$ for local equations of $b$ in $U_{i}$, where $\bar{z}_{1}$ denotes the restriction of $z_{1}$ to $b$. This proves (c) (cf. (0.1)).

$W$ in (d) may be defined as follows. Let $W^{\prime}$ be the plane in $\boldsymbol{P}^{3}$ defined 
by $X_{0}=0$ and let $\bar{W}=W^{\prime} \times A . \bar{W}$ intersects properly with $\mathscr{M}^{\prime}$ on $V^{\prime}$; set $W=\bar{W} \cdot \mathscr{M}^{\prime} . \quad \mu^{-1}$ is biregular at all points of $W$ so regard $W$ as a surface in $\mathscr{M}$. The function $x_{1}=X_{1} / X_{0}$ induced on $\mathscr{M}$ has divisor $T_{\infty}+L_{\infty}$ $-W$. This concludes the proof.

Fix a general quartic surface $B^{\prime}$ in $P^{3}$ and let $X, Y$ be the $K 3$ surfaces determined by $B^{\prime}$ in the manner described in (0.1). Set $k=\operatorname{def}\left(B^{\prime}\right)$.

Proposition 6. (a) There is a variety $\mathscr{K}$ and a proper morphism $f: \mathscr{K}$ $\rightarrow A$, rational over $k$, defining a family of nonsingular $K 3$ surfaces, whose members are as follows. $K_{0}=f^{-1}(0)$ is isomorphic to $Y$ and $K_{x}=f^{-1}(x)$ is isomorphic to $X$ when $x \in A, x \neq 0$. (b) $\mathscr{K}$ carries two pencils of surfaces $\langle\mathscr{T}\rangle$ and $\langle\mathscr{L}\rangle$ such that $\langle\mathscr{T}\rangle$ (resp. $\langle\mathscr{L}\rangle$ ) cuts the pencil $\langle E\rangle$ (resp. the pencil $\left\langle E^{\prime}\right\rangle$ ) on $K_{x}, x \neq 0$, and the pencil $\langle E\rangle+R+\bar{R}$ (resp. the pencil $\langle E\rangle$ ) on $K_{0}$. (c) f factors through the morphism $\varphi$ of the previous Proposition, i.e. there is a morphism $\delta: \mathscr{K} \rightarrow \mathscr{M}$ with $f=\varphi \circ \delta$; and $\delta$ is étale in an open neighborhood of $R$ in $\mathscr{K}$. (d) $\mathscr{K}$ contains a surface $\mathscr{W}$, disjoint from $R$, with $\mathscr{W} \sim \mathscr{T}+\mathscr{L}$ when $\mathscr{T} \in\langle\mathscr{T}\rangle, \mathscr{L} \in\langle\mathscr{L}\rangle$.

Proof. Take $\mathscr{M}, \mathscr{M}^{\prime}$ as in the previous Proposition. Let $D^{\prime}$ be a nonsingular quadric surface in $\boldsymbol{P}^{3}$ over $k$ and define $\bar{B}=B^{\prime} \times A, \bar{D}=D^{\prime}$ $\times A$ in $V^{\prime}=P^{3} \times A$. Then $\bar{B}$ and $\bar{D}$ each intersect properly with $\mathscr{M}^{\prime}$ on $V^{\prime}$; set $B=\bar{B} \cdot \mathscr{M}^{\prime}$ and $D=\bar{D} \cdot \mathscr{M}^{\prime} . \quad \mu^{-1}$ is biregular at all points of $B$ and $D$ so we shall regard them as surfaces in $\mathscr{M}$. Then $B$ and $D$ are irreducible nonsingular surfaces in $\mathscr{M}$ and $B \sim 2 D$. These data determine uniquely a nonsingular, flat, finite double cover $\delta: \mathscr{K} \rightarrow \mathscr{M}$ with branch locus $B$. In fact, $\mathscr{K}=\operatorname{Spec}_{\mathscr{A}}(\mathscr{A})$ where $\mathscr{A}$ is the locally free $\mathcal{O}_{\mathscr{A}}$-algebra $\mathcal{O}_{\mathcal{A}} \oplus \mathcal{O}_{\mathcal{A}}(-D)$ with algebra structure determined by choice of $B$, cf. (0.1).

We have the proper morphism $\varphi: \mathscr{M} \rightarrow A$ of the previous Proposition. Define $f=\varphi \circ \delta$. Then $f$ is a proper morphism and, when $x \in A, f^{-1}(x)$ $=\delta^{-1}\left(\varphi^{-1}(x)\right)$ ([26], p. 240). Write $M_{x}=\varphi^{-1}(x) . \quad \delta$ is a flat morphism, therefore $\mathscr{T}_{\text {or }}{ }^{\mathcal{O}_{i}}\left(\mathcal{O}_{\mathscr{x}}, \delta^{*} \mathcal{O}_{M x}\right)$ vanishes for $i>0$, so the alternating sum of cycles of the sheaves $\mathscr{T}_{\text {oi }_{i}}$ reduces to $\mathscr{K} \times{ }_{k} M_{x}$. Consequently $f^{-1}(x)$ $=\mathscr{K} \times{ }_{\mu} M_{x}$, [24]. Now $\mathscr{K} \times{ }_{\mu} M_{x}=\operatorname{Spec}_{\mathscr{M}}\left(\mathscr{A} \otimes_{O_{M}} \mathcal{O}_{M_{x}}\right)=\operatorname{Spec}_{\mathscr{H}}\left(\mathcal{O}_{M_{x}} \oplus \mathcal{O}_{M_{x}}\right.$ $\left(-D \cdot M_{x}\right)$ ). By construction, $D_{x}=D \cdot M_{x}$ and $B_{x}=B \cdot M_{x}$ are nonsingular curves on $M_{x}$ with $B_{x} \sim 2 D_{x}$. Then $f^{-}(x)=\operatorname{Spec}_{M_{x}}\left(\mathcal{O}_{M_{x}} \oplus \mathcal{O}_{M_{x}}\left(-D_{x}\right)\right)$ and this is the $K 3$ surface as stated in (a), according to (0.1).

$\delta$ is unramified and hence étale, in an open neighborhood of $R$ in $\mathscr{K}$. Define pencils $\langle\mathscr{T}\rangle$ and $\langle\mathscr{L}\rangle$ in $\mathscr{K}$ by setting $\mathscr{T}=\delta^{-1}(T), \mathscr{L}=\delta^{-1}(L)$; 
likewise set $\mathscr{W}=\delta^{-1}(W)$. Then $\mathscr{W} \sim \mathscr{L}+\mathscr{T}$. The counter image of $\mathscr{M}$ cycles by $\delta$ is compatible with intersection-product, as $\delta$ is a morphism, [26], p. 234; hence $\mathscr{T} \cdot K_{x}=\delta^{-1}\left(T \cdot M_{x}\right)$ for $x \in A$, similarly for the members of $\langle\mathscr{L}\rangle$. But $\delta^{-1}\left(T \cdot M_{x}\right)=\delta^{-1}(\ell)=E$ on $K_{x}$ when $x \neq 0$; similarly for the other cases. This concludes the proof.

Proposition 7. Let $f: V \rightarrow V^{\prime}$ be an étale covering (=finite and étale) inducing an isomorphism on subvarieties $W \rightarrow W^{\prime}$. Suppose $V, V^{\prime}, W, W^{\prime}$ are nonsingular; let $N=N_{V / W}, N^{\prime}=N_{V^{\prime} / W^{\prime}}$ be the respective normal bundles. Then $\left(\left.f\right|_{W}\right)^{*} N^{\prime}$ is naturally identified with $N$. Thus $N^{\prime}$ and $N$ are isomorphic as bundles on isomorphic spaces.

Proof. Since $N^{\prime}$ is supported on $W^{\prime},\left(\left.f\right|_{W}\right) * N^{\prime}=N^{\prime} \times{ }_{{ }^{\prime}} W=N^{\prime} \times{ }_{V^{\prime}} W$ $=N^{\prime} \times{ }_{V^{\prime}} V \times{ }_{V} W=\left.\left(f^{*} N^{\prime}\right)\right|_{W}$. Let $\left\{U_{r}^{*}\right\}$ be a finite collection of affine open subsets of $V$, whose union covers $W$ and does not meet the closed set $f^{-1}\left(W^{\prime}\right)-W$. Then $f\left(U_{r}^{*}\right)$ is open in $V^{\prime}([6], I V, 2.4 .6)$ and the union of the $f\left(U_{\gamma}^{*}\right)$ covers $W^{\prime}$. Let $\left\{U_{\alpha}^{\prime}\right\}$ be a finite collection of affine open sets in $V^{\prime}$, whose union covers $W^{\prime}$, such that there are uniformizing parameters $\left\{u_{\alpha 1}^{\prime}, \cdots, u_{\alpha n}^{\prime}\right\}$ in $U_{\alpha}^{\prime}$ with $\left\{u_{\alpha 1}^{\prime}, \cdots, u_{\alpha m}^{\prime}\right\}$ local equations for $W^{\prime}$ in $U_{\alpha}^{\prime}$, for each $\alpha ; n=m+\operatorname{dim} W=\operatorname{dim} V$. Further assume that each $U_{\alpha}^{\prime}$ is contained in some $f\left(U_{r^{(\alpha)}}^{*}\right)$. Let $U_{\alpha}=f^{-1}\left(U_{\alpha}^{\prime}\right) \cap U_{\gamma^{(\alpha)}}^{*}, u_{\alpha i}=f^{*} u_{\alpha i}^{\prime}$. Then each $U_{\alpha}$ is affine and their union covers $W$. Furthermore the $u_{\alpha i}$ are local equations for $W$ in $U_{\alpha}$ : indeed, let $A=\mathcal{O}_{V}\left(U_{\alpha}\right)$ and $p=I\left(W \cap U_{\alpha}\right)$ in $A$, then as $f$ is étale, the $u_{\alpha i}$ generate the localization $p_{m}$ in $A_{m}$ for every maximal ideal $m \supset p$ in $A$, hence they generate $p$ in $A$ (Bourbaki, Alg. Comm. § II.3).

Restriction to $W^{\prime}$ of relations between the $u_{\alpha i}^{\prime}$ and $u_{\beta j}^{\prime}$ with coefficients in $\mathcal{O}_{V^{\prime}}\left(U_{\alpha}^{\prime} \cap U_{\beta}^{\prime}\right)$, determines a system of $m \times m$ matrices $C_{\alpha \beta}^{\prime}$ with coefficients in $\mathcal{O}_{W^{\prime}}\left(U_{\alpha}^{\prime} \cap U_{\beta}^{\prime} \cap W^{\prime}\right)$ defining $N^{\prime}$ on $W^{\prime}$ [21]. The restriction to $W$ of the pullbacks of those relations, determines a system of matrices $C_{\alpha \beta}$ defining $\left.f^{*} N^{\prime}\right|_{W}$. But these same matrices $C_{\alpha \beta}$ also define $N$ because the $u_{\alpha i}$ are local equations for $W$ in $U_{\alpha}$.

Now let $B^{\prime}$ be a general quartic surface in $\boldsymbol{P}^{3}$ over $k_{0}$ and let $p: \mathscr{K}$ $\rightarrow A$ be the family of $K 3$ surfaces determined by $B^{\prime}$ in the manner described in Proposition 6 . Let $R$ be one of the 2 nonextending nodal curves on $Y=p^{-1}(0)$.

TheOREM 2. The elementary operation in $\mathscr{K}$ along $R$ is algebraic. 
Proof. Propositions 6 and 7 show that the normal bundle in $\mathscr{K}$ along $R$ is isomorphic to $\mathcal{O}_{R}(-1) \oplus \mathcal{O}_{R}(-1)$. Then the theorem follows from Propositions 6 and 4 .

3.2. The elementary operation is not algebraic in some cases.

Let $C$ be an irreducible nonsingular curve defined over an algebraically closed field $k$. Let $\mathscr{U} / k$ be a variety and let $p: \mathscr{U} \rightarrow C$ be a proper morphism, rational over $k$, defining an algebraic family of nonsingular non-ruled polarized surfaces $U_{c}$. Suppose that $U_{0}=p^{-1}(0)$ carries a nonextending nodal curve $R$, where 0 is a $k$-rational point of $C$.-For instance, take a general quartic surface in $\boldsymbol{P}^{3}$ and let it specialize in $\boldsymbol{P}^{3}$ so as to acquire a straight line. ${ }^{*}$ - Then, the elementary operation in $\mathscr{U}$ at $R$ cannot be algebraic if the Picard number of $U_{c}$ is 1 when $c$ is a generic point of $C$ over $k$. For, suppose it is algebraic. Then there is a proper morphism $p^{*}: \mathscr{U}^{*} \rightarrow C$ defining an algebraic family of nonsingular surfaces and a birational transformation $\eta: \mathscr{U} \rightarrow \mathscr{U}^{*}$ with $p^{*}=p \circ \eta^{-1},(2.1)$. Moreover let $T$ be the graph of $\eta$. Composition of projection from $T$ with $p$ (or $p^{*}$ ) defines a proper morphism $\tau: T \rightarrow C$ and $T_{c}=\tau^{-1}(c)$ is the graph of the isomorphism induced on $U_{c}$ by $\eta$. But $T_{c}$ specializes to $T_{0}=\tau^{-1}(0)$ and components of $T_{0}$ include $R \times R^{*}$, besides the graph of an isomorphism between $U_{0}$ and $U_{0}^{*},(2.1)$. Therefore, by Theorem 2 in [15], $p^{*}: U^{*} \rightarrow C$ cannot be a family of polarized surfaces. Yet it must be a family of polarized surfaces, according to Matsusaka's theorem on stability of polarization, [14], because $U_{c}^{*} \cong U_{c}$ is a nonsingular algebraic surface with Picard number 1 . The only way out is that the algebraic elementary operation in $\mathscr{U}$ at $R$ does not exist.

The theorem in [14] can be proved rapidly for surfaces using an argument of M. Artin ([1], p. 330), together with the Nakai criterion for ampleness, as follows. Let $p: \mathscr{U} \rightarrow C$ be as above and suppose that when $c$ is a generic point of $C$ over $k, U_{c}=p^{-1}(c)$ has Picard number 1 . Let $X_{c}$ be an effective generator of the divisor class group on $U_{c}$ with respect to numerical equivalence. Let $X_{0}$ be a specialization of $X_{c}$ over $c \rightarrow 0$ with respect to $k$. $X_{0}$ lies on $U_{0}=p^{-1}(0)$; suppose that $U_{0}$ carries an irreducible curve $Y$ with $I\left(X_{0}, Y\right)=0$. $\mathscr{U}$ is an algebraic variety so there is an affine open set $W$ in $\mathscr{U}$ meeting $Y$. Now the complement of $W$ in $\mathscr{U}$ is the support of an effective divisor $D$ on $\mathscr{U}$ (if $P \in \mathscr{U}-D$, then

\footnotetext{
*) This example was shown me by Igor Dolgachev.
} 
$P \in f^{-1}(\infty) \subset \mathscr{U}-D$ for any $f$ in $k[W]$ but not in $\mathcal{O}_{P}$; cf. [5], p. 162). $D$ is not a fibre of the morphism $p$, hence $D$ intersects properly with such fibres; let $D_{0}=D \cdot U_{0}, D_{c}=D \cdot U_{c}$. Then $D_{c}$ must be numerically equivalent to a positive integer multiple of $X_{c}$, say $D_{c} \equiv r X_{c}$, so $D_{0} \equiv r X_{0}$. Therefore $I\left(D_{0}, Y\right)=0$ so $Y$ must be contained in $W$, which is impossible. So we have that $X_{0}$ is effective and $I\left(X_{0}, Y\right)>0$ for every irreducible curve $Y$. Therefore $X_{0}$ defines a polarization on $U_{0}$ and $p: \mathscr{U} \rightarrow C$ is a family of polarized surfaces.

\section{Appendix}

A.1. Here we shall discuss a formal analogue of Horikawa's result [9] about nodal curves, which was mentioned in (2.1). We conserve the terminology and notations of (2.1): $R$ is a nodal curve on a member $S=p^{-1}(0)$ of an algebraic family $p: \mathscr{S} \rightarrow C$ of nonsingular surfaces over a nonsingular curve. It is assumed that the sequence of successive monoidal transformations $\mathscr{S} \leftarrow \mathscr{S}_{1} \leftarrow \mathscr{S}_{2} \leftarrow \cdots$ along nodal curves $R, R_{1}$, $R_{2}, \cdots$ never terminates, so that the normal bundles of $R_{n}$ in $\mathscr{S}_{n}$ are all isomorphic to $\mathcal{O}_{P_{1}} \oplus \mathcal{O}_{P^{1}}(-2)$ (whence the exceptional surfaces $E_{1}, E_{2}, \ldots$ are all copies of the ruled surface $F_{2}$ ). Horikawa's result is that, in the analytic case, $R$ must then extend to a relative divisor, locally in $\mathscr{S}$.

Given $p: \mathscr{S} \rightarrow C, 0 \in C$. Let $t$ be a uniformizing parameter at 0 on $C$ and identify $t$ with the family parameter $t \circ p$ induced on $\mathscr{S}$. We take a finite collection $\left\{U_{i}\right\}$ of affine open sets in $\mathscr{S}$, whose union covers $R$, such that there are uniformizing parameters $\left\{z_{i}, w_{i}, t\right\}$ in $U_{i}$ with $w_{i}=0$, $t=0$ local equations for $R$ in $U_{i}$, for each $i$. Denote by $A_{i}$ the ring $\mathcal{O}_{\mathscr{G}}\left(U_{i}\right)$, by $\mathscr{I}$ the sheaf of ideals of $R$ in $\mathscr{S}$, and by $a_{i}=\mathscr{I}\left(U_{i}\right)=\left(w_{i}, t\right) A_{i}$ the ideal of $R \cap U_{i}$ in $A_{i}$; also, set $A_{i j}=\mathcal{O}_{\mathscr{S}}\left(U_{i} \cap U_{j}\right), a_{i j}=\mathscr{I}\left(U_{i} \cap U_{j}\right)$.

Proposition 8. For each $n, n=0,1,2, \cdots$ there are elements $w_{i}^{(n)}$ in $A_{i}$ as follows. (1) $\left\{z_{i}, w_{i}^{(n)}, t\right\}$ are uniformizing parameters in $U_{i}$ for each $i$, and $w_{i}^{(n)}=0, t=0$ are local equations for $R$ in $U_{i}$. (2) $w_{i}^{(0)} \equiv w_{i}\left(\bmod a_{i}\right)$ and $w_{i}^{(n+1)} \equiv w_{i}^{(n)}\left(\bmod a_{i}^{n+2}\right)$. (3) There are elements $u_{i j}^{(n)}$ in $A_{i j}$ such that $u_{i j}^{(n+1)} \equiv u_{i j}^{(n)}\left(\bmod a_{i j}^{n+2}\right), w_{j}^{(n)}-u_{i j}^{(n)} w_{i}^{(n)} \in a_{i j}^{n+2}$, and $u_{i j}^{(n)}$ is invertible in the local rings $\mathcal{O}_{P}$ on $\mathscr{S}$ for all points $P$ in $R \cap U_{i} \cap U_{j}$.

Proof. For each $n$ we shall define a system of polynomial expressions $P_{i}^{(n)}(t)=\sum_{s=0}^{n} d_{i}^{(s)} t^{s+1}$, of degree $n+1$ in $t$, with coefficients $d_{i}^{(s)}$ in $A_{i}$; observe that by definition $P_{i}^{(n)}-P_{i}^{(n-1)}=d_{i}^{(n)} t^{n+1}$. We set $w_{i}^{(n)}=w_{i}-P_{i}^{(n)}$ 
for each $i$. The $P_{i}^{(n)}$ are to be such that, when $j$ is different from $i, w_{\jmath}^{(n)}$ can be written so:

$$
w_{j}^{(n)}=c_{10} w_{i}^{(n)}+c_{20} w_{i}^{(n) 2}+c_{11} w_{i}^{(n)} t+c_{30} w_{i}^{(n) 3}+\cdots+c_{1 n} w_{i}^{(n)} t^{n}+r_{i j}^{(n)},
$$

where the coefficients $c_{\alpha \beta}$ are elements of $A_{i j}$ (we suppress indices $i, j$ on the $c_{\alpha \beta}$; they are independent of $\left.n\right), r_{i j}^{(n)} \in a_{i j}^{n+2}$, and no terms involving pure powers of $t$ (i.e. no terms $c_{0 \beta} t^{\beta}$ ) of degree $\leqslant n+1$ appear in the expression. Furthermore $c_{10}$ shall not vanish anywhere in $R \cap U_{i} \cap U_{j}$.

When such $P_{i}^{(n)}$ have been found, $w_{i}^{(n)}$ will satisfy conditions (1) and (2), and we may take $u_{i j}^{(n)}$ to be $c_{10}+c_{20} w_{i}^{(n)}+c_{11} t+\cdots+c_{1 n} t^{n}$, so that $w_{j}^{(n)}=u_{i j}^{(n)} w_{i}^{(n)}+r_{i j}^{(n)}$, to conclude the proof. The $P_{i}^{(n)}$ are to be defined inductively, using the hypothesis about the succesive normal bundles on nodal curves $R_{n}$. $n$ is the number of monoidal transformations that have proceeded step $n$ in the induction.

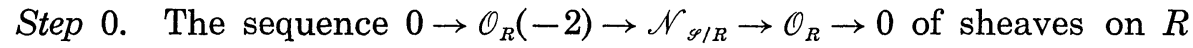
splits by hypothesis; let $g: \mathcal{O}_{R} \rightarrow \mathscr{N}$ be a fixed splitting map. Let $0 \rightarrow$ $\left[\mathcal{O}_{R}(-2)\right] \rightarrow N \stackrel{f}{\rightarrow}\left[\mathcal{O}_{R}\right] \rightarrow 0$ be the corresponding split sequence of vector bundles. The bundles become trivial when restricted to $U_{i} \cap R$, as $R \cong \boldsymbol{P}^{1}$. We let $\omega_{i}$ and $\tau$ be sections of $\left.N\right|_{U_{i} \cap R}$, which are dual to residues of $w_{i}$ and $t$ in $\left.\left(\mathscr{I} / \mathscr{I}^{2}\right)\right|_{U_{i \cap R} \text {. }} \quad \omega_{i}$ and $\tau$ induce bases, and $w_{i}$ and $t$ induce affine coordinate functions, in fibres of $\left.N\right|_{U_{i} \cap R}$. We let $\tau^{\prime}$ denote the global section $f(\tau)$ of $\left[\mathcal{O}_{R}\right]$; note that $f\left(\omega_{i}\right)=0$. Further let $\left(\bar{d}_{i}^{(0)}, 1\right)$ be the local affine coordinates of the section $g\left(\tau^{\prime}\right)$ of $N$ with respect to the basis $\left\{\omega_{i}\right.$, $\tau\}$, where $\bar{d}_{i}^{(0)} \in \mathcal{O}_{R}\left(U_{i} \cap R\right) . \quad U_{i}$ is an affine variety so there is $d_{i}^{(0)}$ in $A_{i}=\mathcal{O}_{\mathscr{S}}\left(U_{i}\right)$ inducing $\bar{d}_{i}^{(0)}$ on $R \cap U_{i}$. We set $P_{i}^{(0)}=d_{i}^{(0)} t$, for each $i$.

We now fix a pair of indices $i, j$. The ideal of the curve $R_{i j}=R \cap U_{i}$ $\cap U_{j}$ in $A_{i j}$ is $\left(w_{i}, t\right) A_{i j}=\left(w_{j}, t\right) A_{i j}=a_{i j}$. Write $w_{j}=c_{10} w_{i}+c_{01} t, c_{\alpha \beta} \in A_{i j}$ (we are suppressing indices $i, j$ on the $c_{\alpha \beta}$; they would otherwise be written $c_{i j}{ }^{\alpha}{ }_{\beta}$ ). $\quad t$ is the family parameter so $w_{i}$ and $w_{j}$ each induce (modulo $t$ ) local equations for $R$ in $S \cap U_{i} \cap U_{j}$, hence $c_{10}$ is an invertible element in the local rings $\mathcal{O}_{P}$ on $\mathscr{S}$ for all points $P$ in $R_{i j}$. Now on the open subset $R_{i j} \subset R$, the transition matrix for the bundle $N$, defining the change from affine coordinates with respect to the basis $\left\{\omega_{i}, \tau\right\}$ to coordinates with respect to the basis $\left\{\omega_{j}, \tau\right\}$ is

$$
\left(\begin{array}{cc}
\bar{c}_{10} & \bar{c}_{01} \\
0 & 1
\end{array}\right),
$$


$\bar{c}_{\alpha \beta}=$ restriction of $c_{\alpha \beta}$ to $R$. Since $g\left(\tau^{\prime}\right)$ is a section of $N,\left(d_{i}^{(0)} c_{10}+c_{01}\right.$ $\left.-d_{j}^{(0)}\right) \in a_{i j}$ so when we set $w_{i}^{(0)}=w_{i}-P_{i}^{(0)}=w_{i}-d_{i}^{(0)} t$ for each $i$ (this defines a natural substitution of coordinate functions), we obtain $w_{j}^{(0)}$ $=c_{10} w_{i}^{(0)}+r_{i j}^{(0)}$, with $r_{i j}^{(0)}=t\left(c_{10} d_{i}^{(0)}+c_{01}-d_{j}^{(0)}\right) \in a_{i j}^{2}$ as required.

Induction Step $(n \geqslant 1)$. Suppose now that we have found polynomials $P_{i}^{(n-1)}=\sum_{s=0}^{n-1} d_{i}^{(s)} t^{s+1}$ and elements $w_{i}^{(n-1)}=w_{i}-P_{i}^{(n-1)}$ in $A_{i}$ such that there is a polynomial expression with remainder for $w_{j}^{(n-1)}$ in terms of $w_{i}^{(n-1)}$ and $t$, viz.

$$
w_{j}^{(n-1)}=c_{10} w_{i}^{(n-1)}+\cdots+c_{\alpha \beta} w_{i}^{(n-1) \alpha} t^{\beta}+\cdots+c_{1 n} w_{i}^{(n-1)} t^{n}+r_{i j}^{(n-1)},
$$

involving no pure powers of $t$ of degree $\leqslant n$, with coefficients $c_{\alpha \beta} \in A_{i j}$ and remainder $r_{i j}^{(n-1)} \in a_{i j}^{n+1}$. Further assume that local equations for $R_{n-1}$ in $U_{i}^{n-1}$ are $w_{i}^{\langle n-1\rangle}=0, t=0$, where $w_{i}^{\langle n-1\rangle} t^{n-1}=w_{i}^{(n-1)}, U_{i}^{0}=U_{i}$, and $U_{i}^{m}$ $(m \geqslant 1)$ is the affine open set in $\mathscr{S}_{m}$ obtained by first pulling back $U_{i}^{m-1}$ to $\mathscr{S}_{m}$ and then deleting points which lie in the proper transform of $E_{m-1}$. We write $r_{i j}^{(n-1)}=c_{0 n+1} t^{n+1}+$ (other homogeneous terms of degree $n+1$ in $w_{i}^{(n-1)}$ and $\left.t\right)+r_{i j}^{[n]}$, with coefficients in $A_{i j}$ and $r_{i j}^{[n]} \in a_{i j}^{n+2}$. Now we blow up $\mathscr{S}_{n-1}$ along $R_{n-1}$; let $U_{i}^{n}$ be the affine open set defined as above. Set $w_{i}^{[n]}=w_{i}^{\langle n-1\rangle} / t=w_{i}^{(n-1)} / t^{n}$. Local equations for $R_{n}$ in $U_{i}^{n}$ are $w_{i}^{[n]}=0, t=0$; furthermore, the $w_{i}^{[n]}$ satisfy the relation

$$
w_{\jmath}^{[n]}=c_{10} w_{i}^{[n]}+c_{0 n+1} t+\cdots+c_{\alpha \beta} w_{\imath}^{[n] \alpha} t^{\beta+\alpha n-n}+\cdots+r_{i j}^{[n]} / t^{n},
$$

when we divide the expression for $w_{j}^{(n-1)}$ by $t^{n}$. Hence the normal bundle of $R_{n}$ in $\mathscr{S}_{n}$ has transition matrix

$$
\left(\begin{array}{ll}
\bar{c}_{10} & \bar{c}_{0 n+1} \\
0 & 1
\end{array}\right)
$$

in $U_{\imath}^{n} \cap U_{j}^{n} \cap R_{n}, \bar{c}_{\alpha \beta}=$ restriction of $c_{\alpha \beta}$ to $R_{n}$. Moreover by hypothesis the sequence $0 \rightarrow \mathcal{O}_{R_{n}}(-2) \rightarrow \mathscr{N}_{\mathscr{S}_{n}} / R_{n} \rightarrow \mathcal{O}_{R_{n}} \rightarrow 0$ of sheaves on $R_{n}$ splits. It is clear by construction that regular functions on the affine curve $R_{n} \cap U_{i}^{n}$ are induced by regular functions on $R \cap U_{i}$, hence by regular functions in $U_{i}$. Then as earlier we find elements $d_{i}^{(n)} \in A_{i}$ with the property that $s^{(n)}=\left(d_{i}^{(n)} c_{10}+c_{0 n+1}-d_{j}^{(n)}\right)$ vanishes on $R_{n} \cap U_{i}^{n} \cap U_{j}^{n}$, and is besides an element of $A_{i j}$. Hence $s^{(n)} \in a_{i j}$. We set $w_{i}^{\langle n\rangle}=w_{i}^{[n]}-d_{i}^{(n)} t$ and find

$$
w_{j}^{\langle n\rangle}=c_{10} w_{i}^{\langle n\rangle}+s^{(n)} t+\cdots+c_{\alpha \beta}\left(w_{i}^{\langle n\rangle}+d_{i}^{(n)} t\right)^{\alpha} t^{\beta+\alpha n-n}+\cdots+r_{i j}^{[n]} / t^{n} .
$$


Put $w_{i}^{(n)}=w_{i}^{\langle n\rangle} t^{n}$ for each $i$ and multiply the expression for $w_{j}^{\langle n\rangle}$ by $t^{n}$. The typical term in the expression becomes $c_{\alpha \beta}\left(w_{i}^{(n)}+d_{i}^{(n)} t^{n+1}\right)^{\alpha} t^{\beta}$; here $\alpha \geqslant 1$ and if $\alpha=1$ then $\beta=1$. Moreover, $s^{(n)} t^{n+1} \in a_{i j}^{n+2}$. Thus we find a polynomial expression

$$
w_{j}^{(n)}=c_{10} w_{\imath}^{(n)}+\cdots+c_{\alpha \beta} w_{i}^{(n) \alpha} t^{\beta}+\cdots+c_{1 n+1} w_{i}^{(n)} t^{n+1}+r_{i j}^{(n)},
$$

involving no pure powers of $t$ of degree $\leqslant n+1$, with coefficients $c_{\alpha \beta} \in A_{i j}$ and remainder $r_{i j}^{(n)} \in a_{i j}^{n+2}$. $w_{\imath}^{\langle n\rangle}=0, t=0$ are local equations for $R_{n}$ in $U_{i}^{n}$. Finally $w_{i}^{(n)}=w_{i}^{(n-1)}-d_{i}^{(n)} t^{n+1}=w_{i}-P_{i}^{(n-1)}-d_{i}^{(n)} t^{n+1}$ so define $P_{i}^{(n)}(t)$ $=P_{i}^{(n-1)}(t)+d_{i}^{(n)} t^{n+1}$. This completes the induction step so the Proposition is proved.

In the analytic case, this proof allows one to apply the arguments of Kodaira [10], [11] to conclude that $R$ extends locally in the family $\mathscr{S}$. Indeed, it shows that "the obstruction $\psi_{m+1}(t)$ vanishes for each integer $m$ ", in the terminology of Kodaira, [11], where we take $d=r=q=1$, $\Phi=N, n=1\left(h^{0}(V, \Phi)=h^{0}(R, N) \geqslant 1\right.$ by our basic hypothesis), $t_{1}=t$, $\beta_{1}=\left(d^{(0)}, 1\right), \varphi^{m}=\left(P^{(m)}, t\right), \psi_{m+1}=\left\{c_{0 m+1} t^{m+1}\right\}$, and $\chi=d^{(m)} t^{m+1}$. Therefore the power series $P_{i}^{*}\left(z_{i}, t\right)=\sum_{s=0}^{\infty} d_{i}^{(s)}\left(z_{i}\right) t^{s+1}$ converge in a small neighborhood of $t=0$, [10], and the local extension of $R$ in $\mathscr{S}$ is defined by holomorphic equations $w_{i}-P_{i}^{*}=0$.

As to the formal case, let $\hat{\mathscr{S}}$ be the completion of $\mathscr{S}$ along $R$ ([6] I, p. 195) and let $w_{\imath}^{*}$ be the element of the $a_{i}$-adic completion $A_{\imath}^{*}=\Gamma\left(U_{i} \cap R\right.$, $\left.\mathcal{O}_{\hat{\varphi}}\right)$ defined by the sequence $\left\{w_{i}^{(n)}\right\}$. Condition 3 of the Proposition shows that the $w_{\imath}^{*}$ define an invertible sheaf $\mathfrak{W}$ of ideals of $\mathcal{O}_{\hat{\varphi}}$, hence a divisor $\Re$ in $\hat{\mathscr{S}}$ ([6] p. 210). Moreover $\left(w_{i}^{*}, t\right) A_{\imath}^{*}=\left(w_{i}, t\right) A_{i}^{*}$ in view of the relation $w_{i}^{*}=w_{i}-P_{i}^{*}$, for each $i$, where $P_{i}^{*}=\sum_{s=0}^{\infty} d_{i}^{(s)} t^{s+1}$ is an element of $t A_{i}^{*}$, by [28] $\S 6$, hence an element of $\left(w_{i}^{*}, t\right) A_{i}^{*} \cap\left(w_{i}, t\right) A_{i}^{*}$. Likewise $\left\{w_{i}^{*}, t\right\}$ is part of a regular system of parameters at every point of $U_{i} \cap R$, so $\Re$ and $S / R$ "cross normally," in the sense of [7] $\S 3$.

A.2. We conserve the terminology and notations of $\S 2.3$. When $R$ is a nonextending nodal curve on $S$ in $\mathscr{S}$ and blowing up along $R$ produces an exceptional surface $E_{1}$ isomorphic to $F_{2}$ instead of $F_{0}$, so that $n \geqslant 2$ in the sequence $\mathscr{S} \leftarrow \mathscr{S}_{1} \leftarrow \cdots \leftarrow \mathscr{S}_{n}$, then apparently stronger assumptions than in Proposition 4 are needed to conclude that the elementary operation in $\mathscr{S}$ along $R$ is algebraic, at least in so far as Lascu's condition $\left(L_{2}\right)$ is concerned. Condition $\left(L_{1}\right)$ is easier:

Suppose that the sequence of monoidal transformations terminates 
after $n$ steps, $n \geqslant 2$, and let $\pi: \mathscr{S}_{n} \rightarrow \mathscr{S}$ be the composition of the transformations. $\pi$ is a proper morphism. Assume that hypothesis (2) in Proposition 4 is satisfied for $\mathscr{S}$. Let $\hat{T}$ be the proper transform of $T$ by $\pi^{-1}$. Then for each $i\left\langle n, \hat{T} \cdot E_{i}\right.$ is a member of the ruling $\langle\ell\rangle$ on the $i^{\text {th }}$ exceptional surface $E_{i} \cong F_{2}$, and $\hat{T} \cdot E_{n}$ is a member of the "other" ruling $\left\langle\ell^{\prime}\right\rangle$ on $E_{n} \cong F_{0}$. Indeed, the intersection-product of $E_{i-1}$ and $E_{i}$ is the nodal curve on the former surface and $\Delta$ on the latter, $i \leqslant n$, so the Corollaries of Proposition 3 apply step by step to successive proper transforms of $T$. Moreover the $\hat{T}$ for $T \in\langle T\rangle$ comprise a linear pencil in $\mathscr{S}_{n}$ with no base points on any $E_{i}$, as we saw in (2.3). Now suppose it is possible to blow down $\mathscr{S}_{n}$ along $E_{n}$ to $\mathscr{S}_{n-1}^{*}$, so that members of $\left\langle\ell^{\prime}\right\rangle$ on $E_{n}$ collapse to points in $\mathscr{S}_{n-1}^{*}$. Let $E_{i}^{*}, \hat{T}^{*}$ denote the transforms of $E_{i}$, $\hat{T}$. Then for each $i, 1 \leqslant i \leqslant n-1, \hat{T}^{*} \cdot E_{i}^{*}$ is a member of the ruling on $E_{i}^{*}$, and the pencil $\left\langle\hat{T}^{*}\right\rangle$ has no base point on any $E_{i}^{*}$. Thus, condition $\left(L_{1}\right)$ of Lascu holds for each $E_{i}^{*}$, when the existence of the pencil $\langle T\rangle$ in $\mathscr{S}$ is assumed.

Condition $\left(L_{2}\right)$ will be satisfied if we make the following assumptions. Suppose that (1) and (2) hold in Proposition 4, and replace (3) by this: (3'): For each $j, 0 \leqslant j \leqslant n-1$, there exists a surface $P_{j} \subset \mathscr{S}$ with $P_{j} \sim L$ $+T, L \in\langle L\rangle, T \in\langle T\rangle$, and such that $P_{0}$ does not meet $R$, all $P_{j}$ with $j \geqslant 1$ pass simply through $R$ on $S$, and successive proper transforms of $P_{j}$ by the $i^{\text {th }}$ monoidal transformation $\pi_{i}^{-1}: \mathscr{S}_{i-1} \rightarrow \mathscr{S}_{i}$, for $1 \leqslant i<j$, pass simply through $R_{i}$ on $E_{i}$ but the proper transform of $P_{j}$ into $\mathscr{S}_{j}$ does not meet $R_{j}$ on $E_{j}$.

Assuming this we have by Proposition 4 that the elementary operation in $\mathscr{S}_{n-1}$ along $R_{n-1}$ is algebraic, as we may take for $P$, in (3) of the Proposition, the proper transform of $P_{n-1}$ into $\mathscr{S}_{n-1}$. Then there is a map $\eta: \mathscr{S}_{n-1} \rightarrow \mathscr{S}_{n-1}^{*} ; \eta$ is biregular everywhere except along $R_{n-1}$ and the (closure of the) graph is everywhere complete over $\mathscr{S}_{n-1}^{*}$. $\eta$ is composed of a blowing up $\pi_{n}^{-1}$ followed by a blowing down $\pi_{n}^{*}$ and induces an isomorphism on $E_{n-1}$, (2.1).

Next we have to dismantle the rest of the scaffolding. Let $\ell$ be a member of the ruling $\langle\ell\rangle_{n-1}$ on $E_{n-1}$ in $S_{n-1}$. We can find $L \in\langle L\rangle, T \in\langle T\rangle$ whose proper transforms $\hat{L}, \hat{T}$ in $\mathscr{S}_{n-1}$ satisfy $(\hat{L}+\hat{T}) \cdot E_{n-1}=2 \ell_{0}+R_{n-1}$, where $\ell_{0}$ is a member of $\langle\ell\rangle_{n-1}$ different from $\ell$. By our assumptions the proper transform $\hat{P}_{n-1}$ of $P_{n-1}$ into $\mathscr{S}_{n-1}$ intersects $E_{n-1}$ in a curve $D$ which does not meet $R_{n-1}$ and is linearly equivalent on $E_{n-1}$ to $2 \ell_{0}+R_{n-1}$; since 
$E_{n-1} \cong F_{2}$, such a $D$ must intersect properly with $\ell$, and must do so with multiplicity one. Moreover there are functions $f$ and $g$ in $k\left(\mathscr{S}_{n-1}\right)$ such that $\operatorname{div}(f)=\hat{P}_{n-1}+E_{n-1}-\hat{P}_{n-2}$ and $\operatorname{div}(g)=\hat{L}+\hat{T}+E_{n-1}-\hat{P}_{n-2}$. Let $f^{*}=f \circ \eta^{-1}, g^{*}=g \circ \eta^{-1}$. We investigate the relations between $\operatorname{div}\left(f^{*}\right)$, $\operatorname{div}\left(g^{*}\right)$, and $E_{n-1}^{*}=\eta\left(E_{n-1}\right) . \quad$ First, $\operatorname{div}\left(f^{*}\right)=\eta(\operatorname{div}(f))$ and $\operatorname{div}\left(g^{*}\right)=\eta(\operatorname{div}(g))$ by ([26], p. 247). By ([26], p. 277, Th. 12b) we have that $\eta(\hat{L})=\pi_{n}^{*}\left(\pi_{n}^{-1}(\hat{L})\right)$, $\eta(\hat{T})=\pi_{n}^{*}\left(\pi_{n}^{-1}(\hat{T})\right)$ and then, by Corollary 1 of Proposition 3 , that $\eta(\hat{L}) \cdot E_{n-1}^{*}$ $=\ell_{0}^{*}+R_{n-1}^{*}, \eta(\hat{T}) \cdot E_{n-1}^{*}=\ell_{0}^{*}$, where $\ell_{0}^{*}$ is $\eta\left(\ell_{0}\right)$. Therefore $E_{n-1}^{*} \cdot\left(\operatorname{div}\left(g^{*}\right)\right.$ $\left.-E_{n-1}^{*}\right)=2 \ell_{0}^{*}+R_{n-1}^{*}$, and $E_{n-1}^{*} \cdot\left(\operatorname{div}\left(f^{*}\right)-E_{n-1}^{*}\right)=\eta(D)$.

Consequently, $f^{*}$ and $g^{*}$ are sections of the ideal of $E_{n-1}^{*}$ over a neighborhood of $\ell^{*}=\eta(\ell)$ in $\mathscr{S}_{n-1}^{*}$ with the properties required by condition $\left(L_{2}\right)$ of Lascu. In this way we blow down all the transforms of $E_{n}, E_{n-1}$, $\cdots, E_{1}$.

\section{REFERENCES}

[1] M. Artin, Algebraic construction of Brieskorn's resolutions, Journal of Algebra 29 (1974), 330-348.

[2] M. F. Atiyah, On analytic surfaces with double points, Proc. Roy. Soc. A247 (1958), 237-244.

[ 3 ] D. Burns, Jr. and M. Rapoport, On the Torelli problem for Kählerian $K 3$ surfaces, Ann. scient. Éc. Norm. Sup, $4^{\text {e }}$ série, t. 8 (1975), 235-274.

[4] I. V. Dolgachev, On special algebraic K3 surfaces, Math. USSR Izvestia vol. 7, No. 4 (1973), 833-846.

[5] J. E. Goodman, Affine open subsets of algebraic varieties and ample divisors, Ann. of Math. 89 (1969), 160-183.

[6 ] A. Grothendieck and J. Dieudonne, Éléments de Géométrie Algébrique, Publ. Math. IHES, nos. 4,.. (1960ff).

[ 7 ] A. Grothendieck and J. P. Murre, The tame fundamental group of a formal neighborhood of a divisor with normal crossings on a scheme, Springer-Verlag, Lecture Notes in Math \#208, Berlin (1971).

[ 8 ] E. Horikawa, On deformations of Quintic Surfaces, Inventiones math. 31 (1975), $43-85$.

[9] — Algebraic surfaces of general type with small $c_{1}{ }^{2}$, II, Inventiones math. 37 (1976), 121-155.

[10] K. Kodaira, A theorem of completeness of characteristic systems for analytic families of compact submanifolds of complex manifolds, Ann. of Math. 75 (1962), 146-162.

[11] —, On stability of compact submanifolds of complex manifolds, Amer. J. Math. 85 (1963), 79-94.

[12] A. T. Lascu, Sous-variété régulièrement contractibles d'une variété algébrique, Ann. Scuola Norm. Sup. Pisa (3) 23 (1969), 675-695.

[13] T. Matsusaka, Algebraic deformations of polarized varieties, Nagoya Math. J. 31 (1968), 185-245.

[14] — On stability of polarization, Number Theory, Alg. Geom. and Comm. Alg., in honor of Y. Akizuki, Tokyo (1973), 495-509. 
[15] T. Matsusaka and D. Mumford, Two fundamental theorems on deformations of polarized varieties, Amer. J. Math. 86 (1964), 668-684.

[16] A. Mayer, Families of K3 surfaces, Nagoya Math. J. 48 (1972), 1-17.

[17] D. Mumford, Introduction to algebraic geometry, Harvard U. Notes (1965).

[18] —-, Algebraic geometry I, Springer-Verlag, Grundlehren der Mathematischen Wissenschaften 221, Berlin (1976).

[19] M. Reid, Hyperelliptic linear systems on a $K 3$ surface, J. London Math. Soc. (2) 13 (1976), 427-437.

[20] B. Saint-Donat, Projective models of $K 3$ surfaces, Amer. J. Math. 96 (1974), 602639.

[21] I. R. Shafarevich, Basic algebraic geometry, Springer-Verlag, Grundlehren der Mathematischen Wissenschaften 213, Berlin (1974).

[22] I. R. Shafarevich et al., Algebraic surfaces, Proc. Steklov Inst. Math. 75 (1965).

[23] P. Samuel, Méthodes d'algèbre abstraite en géométrie algébrique, seconde édition, Springer-Verlag, Ergebnisse der Math. 4, Berlin (1967).

[24] J.-P. Serre, Algèbre locale-multiplicités, Springer-Verlag, Lecture Notes in Math. 11, Berlin (1965).

[25] J. J. Wavrik, Deformations of branched coverings of complex manifolds, Amer. J. Math. 90 (1968), 926-960.

[26] A. Weil, Foundations of Algebraic Geometry, revised edition, Amer. Math. Soc. Publ. 29 (1960).

[27] O. Zariski, Foundations of a general theory of birational correspondences, Trans. Amer. Math. Soc. 53 (1943), 490-542 (+Collected Works, vol. 1, MIT Press).

[28] —- Generalized semi-local rings, Summa Brasiliensis Math. vol. 1, fasc. 8, (1946), 169-195 (+Coll. Works, vol. 2, MIT Press).

[29] — Introduction to the problem of minimal models in the theory of algebraic surfaces, Publ. Math. Soc. Japan 4 (1958) (+Coll. Works, vol. 2, MIT press).

University of Massachusetts at Boston 